\title{
Uniform Attractor for the Fractional Nonautonomous Long-Short Wave Equations
}

\author{
Huanmin Ge and Jie Xin \\ School of Mathematics and Statistics Science, Ludong University, Yantai 264025, China \\ Correspondence should be addressed to Jie Xin; fdxinjie@sina.com
}

Received 14 March 2014; Accepted 3 September 2014; Published 20 October 2014

Academic Editor: Prasanta K. Panigrahi

Copyright ( $) 2014$ H. Ge and J. Xin. This is an open access article distributed under the Creative Commons Attribution License, which permits unrestricted use, distribution, and reproduction in any medium, provided the original work is properly cited.

We firstly proved the existence and the uniqueness of the solution for the $2 \pi$-periodic fractional nonautonomous long-short wave equations with translation compact force by using Galerkin method and then obtained the compact uniform attractor of the system.

\section{Introduction}

In this paper, we consider the following fractional nonautonomous long-short wave equations with translation compact forces:

$$
\begin{gathered}
i u_{t}-(-\Delta)^{\alpha} u-n u+i \delta u=f(x, t), \\
n_{t}+\beta n+|u|_{x}^{2}=g(x, t),
\end{gathered}
$$

with initial and periodic boundary conditions:

$$
\begin{array}{r}
\left.u(x, t)\right|_{t=\tau}=u_{\tau}(x),\left.\quad n(x, t)\right|_{t=\tau}=n_{\tau}(x), \quad x \in \mathbb{R}, \\
u(x+2 \pi, t)=u(x, t), \quad n(x+2 \pi, t)=n(x, t), \\
x \in \mathbb{R}, \quad t \geqslant \tau,
\end{array}
$$

where $u(x, t)$ is an unknown and complex-valued function, $n(x, t)$ is an unknown real valued function, $x \in \Omega=(0,2 \pi) \subset$ $\mathbb{R} ; t \geqslant \tau \in \mathbb{R}^{+} ; \alpha, \delta, \beta>0$, and nonautonomous terms $f(x, t)$ and $g(x, t)$ are time-dependant external forces and translation compact (see Definition 1).

We all know that the long-short wave resonance equations play an important role in fluid mechanics and have rich physical and mathematical properties. There are more and more resent papers treating the long-short wave resonance equations. Guo studied the global solution for one class of the system of LS nonlinear wave interaction in [1] and the periodic initial value problems and initial value problems for one class of generalized long-short type equations in [2].
The papers [3-5] studied the existence of a global attractor of it. Cui et al. developed the weakly compact uniform attractor for the nonautonomous long-short wave equations with translation compact forces in [6].

The Schrödinger type equation has been of great importance describing nonrelativistic quantum mechanical behavior. It is well known that Feynman and Hibbs derive the standard (nonfractional) Schrödinger type equation by applying path integrals over Brownian paths in [7]. Recently Laskin generalized the Schrödinger equation to space fractional cases using path integrals over Lévy trajectories in $[8,9]$. In [10], the authors discussed the models and numerical methods of the fractional calculus. The fractional Schrödinger type equation is used to describe better physical phenomenon and has attracted more and more attention of researchers. Guo and $\mathrm{Xu}$ studied some applications of the Schrödinger equation in physics (see [11]). In [12], the authors obtained the approximate analytical solutions of the fractional nonlinear Schrödinger equations by using the homotopy perturbation method. Eid et al. studied the $\alpha$-dimensional fractional Schrödinger equation and obtained its exact solutions in [13]. Guo et al. investigated the fractional nonlinear Schrödinger equation and showed the existence and uniqueness of its global smooth solution by using energy method in [14]. Goubet [15] studied regularity of the attractor for a weakly damped nonlinear Schrödinger equation in $\mathbb{R}^{2}$.

The rest of this paper is arranged as follows. In Section 2, we recall some basic definitions, introduce preparation results, and analyse some fractional calculation laws which 
depend heavily on $2 \pi$-period. In Section 3 , we introduce some preparation lemma and give the uniform a priori estimates (uniform in initial data and symbol in the symbol space and large time). In Section 4, we show the existence and uniqueness of the solution of the system. In Section 5, we prove the existence of strong compact uniform attractor of the system.

Through the paper, we denote the norm of $L_{\text {per }}^{2}(\Omega)$ with the usual inner product $(\cdot, \cdot)$ by $\|\cdot\|$. We denote the norm of $L_{\text {per }}^{p}(\Omega)$ for all $1<p \leqslant \infty$ by $\|\cdot\|_{L^{p}}$. For simplicity and convenience, the letter $C$ represents a constant, which may be different from one to others. $C(\cdot, \cdot)$ represents the constant $C$ expressed by the parameters appearing in the parentheses.

\section{Preliminaries}

In this section, we introduce notations definition and preliminary facts. We firstly recall the following known definitions (see $[6,16-18])$ and some main lemmas (see $[16,19,20])$.

Definition 1. Suppose $X$ is a Banach space, $f(t): \mathbb{R} \rightarrow X$ is a function, and $T(\cdot)$ is the translation operator. The hull $\mathscr{H}(f)$ of $f$ is defined by

$$
\mathscr{H}(f)=\overline{\{T(s) f(t)=f(t+s) \mid s \in \mathbb{R}\}} .
$$

(i) $f$ is said to be translation bounded in $L^{2}(\mathbb{R} ; X)$ if $\mathscr{H}(f)$ is bounded in which

$$
\|f\|_{L_{b}^{2}(\mathbb{R} ; X)}^{2}:=\sup _{t \in \mathbb{R}} \int_{t}^{t+1}\|f\|_{X}^{2} d s<\infty .
$$

Then $L_{b}^{2}(\mathbb{R} ; X)$ consists of all the translation bounded functions in $L^{2}(\mathbb{R} ; X)$.

(ii) $f$ is called translation compact in $L^{2}(\mathbb{R} ; X)$ if $\mathscr{H}(f)$ is compact in $L^{2}(\mathbb{R} ; X)$, where the convergence is taken in the sense of local quadratic mean convergence topology of $L_{\text {loc }}^{2}\left(\mathbb{R} ; H^{\alpha}\right)$. The collection of all the translation compact functions in $L^{2}(\mathbb{R} ; X)$ is denoted by $L_{c}^{2}(\mathbb{R} ; X)$.

Let $\left(X,\|\cdot\|_{X}\right)$ be a Banach space, and the following definitions are common.

Definition 2. Let $\Sigma$ be a parameter set. $\left\{U_{\sigma}(t, \tau), t \geqslant \tau, \tau \in \mathbb{R}\right\}$, $\sigma \in \Sigma$ is said to be a family of processes in $X$, if, for each $\sigma \in$ $\Sigma, U_{\sigma}(t, \tau)$ is a process; that is, the two-parameter mapping $U_{\sigma}(t, \tau)$ from $X$ to $X$ satisfies

(i) $U_{\sigma}(t, s) \circ U_{\sigma}(s, \tau)=U_{\sigma}(t, \tau), \forall t \geqslant s \geqslant \tau, \tau \in \mathbb{R}$,

(ii) $U_{\sigma}(\tau, \tau)=I$ (the identity operator on $X$ ), $\tau \in \mathbb{R}$,

where $\Sigma$ is called the symbol space and $\sigma \in \Sigma$ is called the symbol.

A subset $B_{0} \subset E$ is said to be uniformly absorbing set for the family of processes $\left\{U_{\sigma \in \Sigma}(t, \tau)\right\}$, if, for any $\tau \in \mathbb{R}$ and subset $B \in \mathscr{B}(E)$ denoting the set of all bounded subsets of $E$, there exists $t_{0}=t_{0}(\tau, B) \geqslant \tau$ such that $\bigcup_{\sigma \in \Sigma} U_{\sigma}(t, \tau) B \subseteq B_{0}$ for all $t \geqslant t_{0}$. A set $Y \subset E$ is called uniformly attracting for the family of process $\left\{U_{\sigma}(t, \tau)\right\}, \sigma \in \sum$ if, for each fixed $\tau \in \mathbb{R}$ and every $B \in \mathscr{B}(E)$, it satisfies that

$$
\lim _{t \rightarrow+\infty}\left(\operatorname{supdist}_{\sigma \in \sum}\left(U_{\sigma}(t, \tau) B, Y\right)\right)=0 .
$$

Definition 3. A closed set $\mathscr{A}_{\Sigma} \subset X$ is called the uniform attractor of the family of processes $\left\{U_{\sigma}(t, \tau)\right\}_{\sigma \in \Sigma}$ if it is uniformly attracting (attracting property) and it is contained in any closed uniformly attracting set $\mathscr{A}^{\prime}$ of the family of processes $\left\{u_{\sigma}(t, \tau)\right\}_{\sigma \in \Sigma}: \mathscr{A}_{\Sigma} \subseteq \mathscr{A}^{\prime}$ (minimality property).

Definition 4. $\left\{U_{\sigma \in \Sigma}(t, \tau)\right\}$, a family of processes in $X$, is said to be $(X \times \Sigma, X)$-continuous, if, for any fixed $T$ and $\tau, T \geqslant \tau$, projection $\left(u_{\tau}, \sigma\right) \rightarrow U_{\sigma}(T, \tau) u_{\tau}$ is continuous from $X \times \Sigma$ to X.

Definition 5. The space $L^{p}(0, T ; X)$ denotes all measurable functions $f:[0, T] \rightarrow X$ with the norm

$$
\|f\|_{L^{p}(0, T ; X)}=\left(\int_{0}^{T}\|f\|_{X}^{p} d t\right)^{1 / p}<\infty
$$

for $1 \leqslant p<\infty$, and

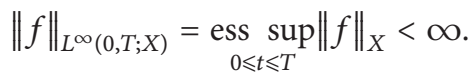

Lemma 6. Let $\sum$ be a compact metric space and suppose $\{T(h) \mid h \geqslant 0\}$ is a family of operators defined on $\sum$, satisfying

$$
T(h) \sum=\sum, \forall h \in \mathbb{R}_{+}
$$

(ii) translation identity:

$$
\begin{array}{r}
U_{\sigma}(t+h, \tau+h)=U_{T(h) \sigma}(t, \tau), \quad \forall \sigma \in \sum, t \geqslant \tau, \\
\tau \in \mathbb{R}, \quad h \geqslant 0,
\end{array}
$$

where $U_{\sigma}(T, \tau)$ is an arbitrary process in compact metric space $E$. Note that if the family of processes $\left\{U_{\sigma \in \Sigma}(T, \tau)\right\}$ is $\left(E \times \sum, E\right)$ continuous and it has a uniform compact attracting set, then the skew product flow corresponding to it has a global attractor $\mathscr{A}$ on $E \times \sum$. And the projection of $\mathscr{A}$ on $\sum, \mathscr{A}_{\sum}$, is the compact uniform attractor of $\left\{U_{\sigma \in \Sigma}(T, \tau)\right\}$.

Remark 7. Assumption (11) holds if the system has a unique solution.

Lemma 8. Let $\left(X,\|\cdot\|_{X}\right)$ be a uniform convex Banach space (particularly, a Hilbert space), and let $\left\{x_{k}\right\}_{k \geqslant 0}$ be a sequence in $X$. If $x_{k} \rightarrow x_{0}$ and $\left\|x_{k}\right\|_{X} \rightarrow\left\|x_{0}\right\|_{X}$, then $x_{k} \rightarrow x_{0}$.

Lemma 9. Let $\left\{x_{k}\right\}_{k \geqslant 0}$ be a sequence in $B^{*}$ space $X$. If $x_{k} \rightarrow$ $x_{0}$, then

$$
\sup _{k \geqslant 1}\left\|x_{k}\right\|_{X}<\infty, \quad\left\|x_{0}\right\|_{X} \leqslant \liminf _{k \rightarrow \infty}\left\|x_{k}\right\|_{X} .
$$


Since the solution $u(x, t)$, if it exists, is a $2 \pi$-periodic function, we have the Fourier expansion:

$$
u(x, t)=\sum_{k \in \mathbb{Z}} a_{k}(t) e^{i k x}
$$

where $a_{k}(t): \mathbb{R}^{+} \rightarrow \mathbb{R}, x \in \mathbb{R}$. Therefore,

$$
\partial_{x} u=\sum_{k \in \mathbb{Z}} i k a_{k} e^{i k x}
$$

and $(-\Delta)^{\alpha} u$ is defined by

$$
(-\Delta)^{\alpha} u=\sum_{k \in \mathbb{Z}}|k|^{2 \alpha} a_{k} e^{i k x}
$$

Since

$$
\left|e^{i r x}\right|^{2}=1, \quad \int_{\Omega} e^{i r x} d x=0, \quad \forall r \in \mathbb{R}
$$

the following definitions make sense. Let

$$
A=\left\{\left.u\left|u=\sum_{k \in \mathbb{Z}} a_{k} e^{i k x}, \sum_{k \in \mathbb{Z}}\right| k\right|^{4 \alpha} a_{k}^{2}<\infty, \sum_{k \in \mathbb{Z}}\left|a_{k}\right|^{2}<\infty\right\},
$$

and let $H^{2 \alpha}$ be a complete space of the set $A$ under the norm:

$$
\begin{aligned}
\|u\|_{H^{2 \alpha}} & =\left\|(-\Delta)^{\alpha} u\right\|+\|u\| \\
& =\left(2 \pi \sum_{k \in \mathbb{Z}}|k|^{4 \alpha} a_{k}^{2}\right)^{1 / 2}+\left(2 \pi \sum_{k \in \mathbb{Z}}\left|a_{k}\right|^{2}\right)^{1 / 2} .
\end{aligned}
$$

Then we can easily check that $H^{2 \alpha}$ is a Banach space and that, for $\forall u \in H^{2 \alpha}$, $u$ is space-periodic with the period $2 \pi$ and its $2 \alpha$ order derivatives are in $L_{\text {per }}^{2}(\Omega)$. And for $\forall u, v \in H^{\alpha}$,

$$
\begin{gathered}
\frac{d}{d t} u v=u \frac{d}{d t} v+v \frac{d}{d t} u, \\
\left((-\Delta)^{\alpha} u, v\right)=\left((-\Delta)^{\alpha_{1}} u,(-\Delta)^{\alpha_{2}} v\right),
\end{gathered}
$$

when $\alpha_{1}+\alpha_{2}=\alpha, 0 \leqslant \alpha_{1}, \alpha_{2} \leqslant \alpha . H^{2 \alpha}$ is a Hilbert space with the inner product

$$
(u, v)_{H^{2 \alpha}}=\left((-\Delta)^{\alpha} u,(-\Delta)^{\alpha} v\right)=(2 \pi)^{n} \sum_{k \in \mathbb{Z}^{n}}|k|^{4 \alpha} a_{k} b_{k} .
$$

For brevity, we introduce $W(x, t)=(u(x, t), n(x, t))$ and $Y(x, t)=(f(x, t), g(x, t))$. We denote the space of functions $W(x, t)=(u(x, t), n(x, t))$ by $E_{0}=H^{2 \alpha}(\Omega) \cap H_{p e r}^{\alpha}(\Omega) \times$ $H_{\text {per }}^{1}(\Omega)$ with norm

$$
\|W\|_{E_{0}}=\left(\|u\|_{H^{2 \alpha}}^{2}+\|n\|_{H^{1}}^{2}\right)^{1 / 2} .
$$

Similarly, we denote the space of $Y(x, t)$ by $\sum_{0}$ with norm

$$
\|Y\|_{\sum_{0}}=\left(\|f\|_{H^{2 \alpha}}^{2}+\|g\|_{H^{1}}^{2}\right)^{1 / 2} \text {. }
$$

Assumption 10. Suppose that the symbol $Y(x, t)$ belongs to the symbol space $\sum$, defined by

$$
\sum=\overline{\left\{Y_{0}(x, s+r) \mid r \in \mathbb{R}_{+}\right\}}
$$

where $Y_{0}=\left(f_{0}(x, t), g_{0}(x, t)\right) \in L_{c}^{2}\left(\mathbb{R} ; E_{0}\right)$ and the closure is taken in the sense of local quadratic mean convergence topology in the topological space $L_{\text {loc }}^{2}\left(\mathbb{R} ; \sum_{0}\right)$. Moreover, we assume $f_{0 t}(x, t) \in L_{b}^{2}\left(\mathbb{R} ; H^{\alpha}\right)$.

Remark 11. Due to the conception of translation compact/boundedness, we remark that

(i) $\forall Y_{1} \in \sum,\left\|Y_{1}\right\|_{L_{b}^{2}\left(\mathbb{R} ; \Sigma_{0}\right)}^{2} \leqslant\left\|Y_{0}\right\|_{L_{b}^{2}\left(\mathbb{R} ; \sum_{0}\right)}^{2}$;

(ii) $T(t) \sum=\sum, \forall t \in \mathbb{R}$, where $T(t) \varphi(s)=\varphi(s+t)$ is a translation operator.

\section{A Uniform A Priori Estimates}

In this section, we will get some uniform a priori estimates which hold uniformly independently of initial data, time, and symbols in symbol space $(Y \in \Sigma)$. In the following, we denote that $\int \cdot d x=\int_{\Omega} \cdot d x$ and $H=L^{2}(\Omega)$, which will not cause any confusions.

We first recall the Gagliardo-Nirenberg and the Young inequalities (see [21]).

Lemma 12. Let $u \in L^{q}\left(\mathbb{R}^{n}\right), D^{m} u \in L^{r}\left(\mathbb{R}^{n}\right), 1 \leqslant q, r<\infty$. Then for $0 \leqslant j \leqslant m, j / m \leqslant a \leqslant 1$, there exists a constant $C$ such that

$$
\left\|D^{j} u\right\|_{L^{p}} \leqslant C\left\|D^{m} u\right\|_{L^{r}}^{a}\|u\|_{L^{q}}^{1-a}
$$

where $1 / p=j / n+a(1 / r-m / n)+(1-a)(1 / q)$.

Lemma 13. Let $a, b>0$. Then for each $(p, q)$ satisfying $1<$ $p, q<\infty, 1 / p+1 / q=1$, it holds that

$$
a \cdot b \leqslant \varepsilon \frac{a^{p}}{p}+\varepsilon^{-q / p} \frac{b^{q}}{q}, \quad \forall \varepsilon>0 .
$$

Lemma 14. Assume that

(i) $Y(x, t)$ satisfy Assumption 10;

(ii) $u_{\tau} \in L^{2}(\Omega)$ and $u(t)$ solves problem $(1)-(4)$.

Then there exist positive constants $C_{1}=C_{1}\left(\delta,\left\|f_{0}\right\|_{L_{b}^{2}\left(R ; H^{2 \alpha}\right)}\right)$ and $t_{1}=t_{1}\left(\delta,\left\|f_{0}\right\|_{L_{b}^{2}\left(R ; H^{2 \alpha}\right)},\left\|u_{\tau}\right\|\right)$ such that

$$
\|u(t)\|^{2} \leqslant C_{1}, \quad \forall t \geqslant t_{1} .
$$

Proof. Taking the inner product of (1) with $u$, we have

$$
\left(i u_{t}-(-\Delta)^{\alpha} u-n u+i \delta u, u\right)=(f(x, t), u) .
$$


Taking the imaginary part of (27), we get

$$
\frac{1}{2} \frac{d}{d t}\|u\|^{2}+\delta\|u\|^{2}=\operatorname{Im}(f(x, t), u) .
$$

By (28) and Remark 11, we have

$$
\begin{aligned}
\frac{d}{d t}\|u\|^{2}+2 \delta\|u\|^{2} & \\
& \leqslant \delta\|u\|^{2}+\frac{1}{\delta}\|f(x, t)\|_{L_{b}^{2}\left(R ; H^{2 \alpha}\right)}^{2} \\
& \leqslant \delta\|u\|^{2}+\frac{1}{\delta}\left\|f_{0}\right\|_{L_{b}^{2}\left(R ; H^{2 \alpha}\right)}^{2} .
\end{aligned}
$$

By Gronwall inequality we get the lemma.

Lemma 15. Assume that

(i) $\alpha \geqslant 1$, and $Y(x, t)$ satisfy Assumption 10;

(ii) $W(\tau) \in H^{\alpha} \times H$ and $W(t)$ solves problem (1)-(4).

Then there exist positive constants $C_{2}=$ $C_{2}\left(\alpha, \delta, \beta,\left\|Y_{0}\right\|_{L_{b}^{2}\left(R ; \Sigma_{0}\right)},\left\|f_{0 t}\right\|_{L_{b}^{2}\left(R ; H^{\alpha}\right)}\right)$ and $t_{2}=t_{2}(\alpha, \delta, \beta$, $\left.\left\|Y_{0}\right\|_{L_{b}^{2}\left(R ; \Sigma_{0}\right)},\left\|f_{0 t}\right\|_{L_{b}^{2}\left(R ; H^{\alpha}\right)},\left\|W_{\tau}\right\|_{H^{\alpha} \times H}\right)$ such that

$$
\|W(t)\|_{H^{\alpha} \times H} \leqslant C_{2}, \quad \forall t \geqslant t_{2} .
$$

Proof. Taking the inner product of (1) with $u_{t}$, we have

$$
\left(i u_{t}-(-\Delta)^{\alpha} u-n u+i \delta u, u_{t}\right)=\left(f(x, t), u_{t}\right) .
$$

Taking the real part of (31), we have

$$
\begin{aligned}
& -\frac{1}{2} \frac{d}{d t}\left\|(-\Delta)^{\alpha / 2} u\right\|-\frac{1}{2} \int n \frac{d}{d t}|u|^{2} d x+\operatorname{Re}\left(i \delta u, u_{t}\right) \\
& =\operatorname{Re}\left(f(x, t), u_{t}\right),
\end{aligned}
$$

where

$$
\begin{aligned}
\int n \frac{d}{d t}|u|^{2} \\
=\int \frac{d}{d t}\left(n|u|^{2}\right) d x-\int|u|^{2} n_{t} d x \\
=\int \frac{d}{d t}\left(n|u|^{2}\right) d x-\int|u|^{2}\left(g(x, t)-\beta n-|u|_{x}^{2}\right) d x \\
=\int \frac{d}{d t}\left(n|u|^{2}\right) d x+\int|u|^{2}|u|_{x}^{2} d x \\
\quad+\beta \int n|u|^{2} d x-\int g(x, t)|u|^{2} d x \\
=\int \frac{d}{d t}\left(n|u|^{2}\right) d x+\beta \int n|u|^{2} d x-\int g(x, t)|u|^{2} d x
\end{aligned}
$$

So we have

$$
\begin{gathered}
\frac{1}{2} \frac{d}{d t}\left(\left\|(-\Delta)^{\alpha / 2} u\right\|^{2}+\int n|u|^{2} d x+2 \operatorname{Re}(f(x, t), u)\right) \\
+\frac{\beta}{2} \int n|u|^{2} d x-\frac{1}{2} \int g(x, t)|u|^{2} d x \\
-\operatorname{Re}\left(i \delta u, u_{t}\right)-\operatorname{Re}\left(f_{t}(x, t), u\right)=0 .
\end{gathered}
$$

Taking the inner product of (1) with $\delta u$, we have

$$
\left(i u_{t}-(-\Delta)^{\alpha} u-n u+i \delta u, \delta u\right)=(f(x, t), \delta u) .
$$

Taking the real part of (35), we have

$$
\begin{aligned}
& \operatorname{Re}\left(i \delta u, u_{t}\right)+\delta\left\|(-\Delta)^{\alpha / 2} u\right\| \\
& \quad+\int \delta n|u|^{2} d x+\operatorname{Re}(f(x, t), \delta u)=0 .
\end{aligned}
$$

By (34) and (36), we have

$$
\begin{aligned}
& \frac{d}{d t}\left(\left\|(-\Delta)^{\alpha / 2} u\right\|^{2}+\int n|u|^{2} d x+2 \operatorname{Re}(f(x, t), u)\right) \\
& \quad+\delta\left(\left\|(-\Delta)^{\alpha / 2} u\right\|^{2}+\int n|u|^{2} d x+2 \operatorname{Re}(f(x, t), u)\right) \\
& \quad+\delta\left\|(-\Delta)^{\alpha / 2} u\right\|^{2} \\
& =\int g(x, t)|u|^{2} d x-(\delta+\beta) \int n|u|^{2} d x \\
& \quad+2 \operatorname{Re}\left(f_{t}(x, t), u\right) .
\end{aligned}
$$

By Lemmas 12 and 13 and the condition $\alpha \geqslant 1$, we have

$$
\int g(x, t)|u|^{2} d x
$$

$$
\begin{aligned}
\leqslant & \frac{1}{2}\left(\|g(x, t)\|^{2}+\|u\|_{L^{4}}^{4}\right) \\
\leqslant & \frac{1}{2}\|g(x, t)\|_{L_{b}^{2}\left(R ; \Sigma_{0}\right)}^{2} \\
& +C\left\|(-\Delta)^{\alpha / 2} u\right\|^{1 / \alpha}\|u\|^{4-(1 / \alpha)} \\
\leqslant & \rho\left\|(-\Delta)^{\alpha / 2} u\right\|^{2} \\
& +C\left(\rho,\left\|Y_{0}\right\|_{L_{b}^{2}\left(R ; \Sigma_{0}\right)}\right),
\end{aligned}
$$

$$
\begin{aligned}
\mid-(\delta & +\beta) \int n|u|^{2} d x \mid \\
& \leqslant \rho\|n\|^{2}+C(\delta, \beta)\|u\|_{L^{4}}^{4} \\
& \leqslant \rho\|n\|^{2}+\rho\left\|(-\Delta)^{\alpha / 2} u\right\|^{2}+C(\rho, \delta, \beta) .
\end{aligned}
$$


So we reduce that

$$
\begin{aligned}
& \frac{d}{d t}\left(\left\|(-\Delta)^{\alpha / 2} u\right\|^{2}+\int n|u|^{2} d x+2 \operatorname{Re}(f(x, t), u)\right) \\
& \quad+\delta\left(\left\|(-\Delta)^{\alpha / 2} u\right\|^{2}+\int n|u|^{2} d x+2 \operatorname{Re}(f(x, t), u)\right) \\
& \quad+\delta\left\|(-\Delta)^{\alpha / 2} u\right\|^{2} \\
& \leqslant \rho\|n\|^{2}+2 \rho\left\|(-\Delta)^{\alpha / 2} u\right\|^{2} \\
& \quad+C\left(\rho,\left\|f_{0 t}\right\|_{L_{b}^{2}\left(R ; H^{\alpha}\right)},\left\|Y_{0}\right\|_{L_{b}^{2}\left(R ; \Sigma_{0}\right)}\right) .
\end{aligned}
$$

Similarly, we also derive that

$$
\begin{aligned}
& \frac{d}{d t}\left(\left\|(-\Delta)^{\alpha / 2} u\right\|^{2}+\int n|u|^{2} d x+2 \operatorname{Re}(f(x, t), u)\right) \\
& \quad+\beta\left(\left\|(-\Delta)^{\alpha / 2} u\right\|^{2}+\int n|u|^{2} d x+2 \operatorname{Re}(f(x, t), u)\right) \\
& \quad+(2 \delta-\beta)\left\|(-\Delta)^{\alpha / 2} u\right\|^{2} \\
& \leqslant \rho\|n\|^{2}+2 \rho\left\|(-\Delta)^{\alpha / 2} u\right\|^{2} \\
&+C\left(\rho,\left\|f_{0 t}\right\|_{L_{b}^{2}\left(R ; H^{\alpha}\right)},\left\|Y_{0}\right\|_{L_{b}^{2}\left(R ; \Sigma_{0}\right)}\right) .
\end{aligned}
$$

Taking the inner product of (2) with $n$, we have

$$
\frac{1}{2} \frac{d}{d t}\|n\|^{2}+\int n|u|_{x}^{2} d x+\beta\|n\|^{2}-\int g(x, t) n d x=0 .
$$

By (1), we have

$$
\begin{aligned}
\int n|u|_{x}^{2} d x & \\
= & \int n(u \bar{u})_{x}=\int\left(n u \bar{u}_{x}+n \bar{u} u_{x}\right) d x \\
= & \int\left(i u_{t}-(-\Delta)^{\alpha} u+i \delta u-f(x, t)\right) \bar{u}_{x} d x \\
& +\int\left(-i \bar{u}_{t}-(-\Delta)^{\alpha} \bar{u}-i \delta \bar{u}-\overline{f(x, t)}\right) u_{x} d x \\
= & i \int\left(u_{t} \bar{u}_{x}-\bar{u}_{t} u_{x}\right) d x+2 \operatorname{Re} \int i \delta u \bar{u}_{x} d x \\
& -2 \operatorname{Re} \int f(x, t) \bar{u}_{x} d x,
\end{aligned}
$$

where

$$
\begin{aligned}
& \frac{d}{d t} \int\left(i u \bar{u}_{x}-i u_{x} \bar{u}\right) d x \\
& \quad=i \int\left(u_{t} \bar{u}_{x}+u \bar{u}_{x t}-u_{x t} \bar{u}-u_{x} \bar{u}_{t}\right) d x \\
& \quad=i \int\left(u_{t} \bar{u}_{x}-u_{x} \bar{u}_{t}+u_{t} \bar{u}_{x}-u_{x} \bar{u}_{t}\right) d x \\
& \quad=2 i \int\left(u_{t} \bar{u}_{x}-u_{x} \bar{u}_{t}\right) d x .
\end{aligned}
$$

By (41) (43), we get

$$
\begin{aligned}
\frac{d}{d t}\left(\|n\|^{2}\right. & \left.+\int i\left(u \bar{u}_{x}-u_{x} \bar{u}\right) d x\right)+2 \beta\|n\|^{2} \\
& +\delta \int i\left(u \bar{u}_{x}-u_{x} \bar{u}\right) d x \\
= & \delta \int i\left(u \bar{u}_{x}-u_{x} \bar{u}\right) d x-4 \operatorname{Re} \int i \delta u \bar{u}_{x} d x \\
& +4 \operatorname{Re} \int f(x, t) \bar{u}_{x} d x+2 \int g(x, t) n d x \\
= & -\delta \int i\left(u \bar{u}_{x}-u_{x} \bar{u}\right) d x+4 \operatorname{Re} \int f(x, t) \bar{u}_{x} d x \\
& +2 \int g(x, t) n d x .
\end{aligned}
$$

By Lemmas 12 14 and the condition $\alpha \geqslant 1$, we have

$$
\begin{aligned}
& \left|i \delta \int\left(u \bar{u}_{x}-u_{x} \bar{u}\right) d x\right| \leqslant 2 \delta\|u\|\left\|u_{x}\right\| \\
& \leqslant \rho\left\|(-\Delta)^{\alpha / 2} u\right\|^{2}+C(\rho), \\
& \left|4 \operatorname{Re} \int f(x, t) \bar{u}_{x} d x\right| \leqslant 4\|f(x, t)\|\left\|\bar{u}_{x}\right\| \\
& \leqslant \rho\left\|(-\Delta)^{\alpha / 2} u\right\|^{2}+C\left(\rho,\left\|Y_{0}\right\|_{L_{b}^{2}\left(R ; \Sigma_{0}\right)}\right), \\
& \left|2 \int g(x, t) n d x\right| \leqslant 2\|g(x, t)\|\|n\| \\
& \leqslant \rho\|n\|^{2}+C\left(\rho,\left\|Y_{0}\right\|_{L_{b}^{2}\left(R ; \Sigma_{0}\right)}\right) .
\end{aligned}
$$

So we obtain that

$$
\begin{aligned}
\frac{d}{d t}\left(\|n\|^{2}\right. & \left.+\int i\left(u \bar{u}_{x}-u_{x} \bar{u}\right) d x\right) \\
& +\delta\left(\|n\|^{2}+\int i\left(u \bar{u}_{x}-u_{x} \bar{u}\right) d x\right) \\
& +\beta\|n\|^{2}+(\beta-\delta)\|n\|^{2} \\
= & \rho\|n\|^{2}+2 \rho\left\|(-\Delta)^{\alpha / 2} u\right\|^{2}+C\left(\rho,\left\|Y_{0}\right\|_{L_{b}^{2}\left(R ; \Sigma_{0}\right)}\right)
\end{aligned}
$$

Similarly, we can also get that

$$
\begin{aligned}
\frac{d}{d t}\left(\|n\|^{2}\right. & \left.+\int i\left(u \bar{u}_{x}-u_{x} \bar{u}\right) d x\right) \\
& +\beta\left(\|n\|^{2}+\int i\left(u \bar{u}_{x}-u_{x} \bar{u}\right) d x\right)+\beta\|n\|^{2} \\
= & \rho\|n\|^{2}+2 \rho\left\|(-\Delta)^{\alpha / 2} u\right\|^{2}+C\left(\rho,\left\|Y_{0}\right\|_{L_{b}^{2}\left(R ; \Sigma_{0}\right)}\right) .
\end{aligned}
$$

Set

$$
\begin{aligned}
E= & \left\|(-\Delta)^{\alpha / 2} u\right\|^{2}+\|n\|^{2}+\int n|u|^{2} d x+2 \operatorname{Re}(f(x, t), u) \\
& +\int i\left(u \bar{u}_{x}-u_{x} \bar{u}\right) d x .
\end{aligned}
$$


So by (39) and (46), we get

$$
\begin{aligned}
\frac{d}{d t} E+ & \delta E+\delta\left\|(-\Delta)^{\alpha / 2} u\right\|^{2}+\beta\|n\|^{2}+(\beta-\delta)\|n\|^{2} \\
\leqslant & 4 \rho\left\|(-\Delta)^{\alpha / 2} u\right\|^{2}+2 \rho\|n\|^{2} \\
& +C\left(\rho,\left\|f_{0 t}\right\|_{L_{b}^{2}\left(R ; H^{\alpha}\right)},\left\|Y_{0}\right\|_{L_{b}^{2}\left(R ; \Sigma_{0}\right)}\right) .
\end{aligned}
$$

By (40) and (47), we also get

$$
\begin{aligned}
\frac{d}{d t} E+ & \beta E+\delta\left\|(-\Delta)^{\alpha / 2} u\right\|^{2}+\beta\|n\|^{2}+(\delta-\beta)\left\|(-\Delta)^{\alpha / 2} u\right\|^{2} \\
\leqslant & 4 \rho\left\|(-\Delta)^{\alpha / 2} u\right\|^{2}+2 \rho\|n\|^{2} \\
& +C\left(\rho,\left\|f_{0 t}\right\|_{L_{b}^{2}\left(R ; H^{\alpha}\right)},\left\|Y_{0}\right\|_{L_{b}^{2}\left(R ; \Sigma_{0}\right)}\right) .
\end{aligned}
$$

Setting $\gamma=\min \{\delta, \beta\}, \rho \leqslant \min \{\delta / 4, \beta / 2\}$, then we deduce that

$$
\frac{d}{d t} E+\gamma E \leqslant C\left(\rho,\left\|Y_{0}\right\|_{L_{b}^{2}\left(R ; \Sigma_{0}\right)},\left\|f_{0 t}\right\|_{L_{b}^{2}\left(R ; H^{\alpha}\right)}\right)=C_{0}, \quad \forall t \geqslant t_{0} .
$$

By Gronwall inequality, we have

$$
E(t) \leqslant E\left(t_{0}\right) e^{-\gamma\left(t-t_{0}\right)}+\frac{C_{0}}{\gamma}, \quad \forall t \geqslant t_{0} .
$$

Obviously for any $t \geqslant t_{0}$, we have

$$
\begin{aligned}
& \left.\left|\int n\right| u\right|^{2} d x+2 \operatorname{Re}(f(x, t), u)+\int i\left(u \bar{u}_{x}-u_{x} \bar{u}\right) d x \mid \\
& \leqslant \rho\|n\|^{2}+\rho\left\|(-\Delta)^{\alpha / 2} u\right\|^{2}+C\left(\rho,\left\|Y_{0}\right\|_{L_{b}^{2}\left(R ; \Sigma_{0}\right)}\right), \\
& \left|E\left(t_{0}\right)\right| \leqslant\left\|(-\Delta)^{\alpha / 2} u\left(t_{0}\right)\right\|^{2}+\left\|n\left(t_{0}\right)\right\|^{2} \\
& +\left.\left|\int n\left(t_{0}\right)\right| u\left(t_{0}\right)\right|^{2} d x+2 \operatorname{Re}\left(f\left(t_{0}\right), u\left(t_{0}\right)\right) \\
& +\int i\left(u\left(t_{0}\right) \bar{u}_{x}\left(t_{0}\right)-u_{x}\left(t_{0}\right) \bar{u}\left(t_{0}\right)\right) d x \mid \\
& \leqslant C\left(\rho,\left\|Y_{0}\right\|_{L_{b}^{2}\left(R ; \Sigma_{0}\right)},\left\|W_{\tau}\right\|_{H^{\alpha} \times H}\right) .
\end{aligned}
$$

So by $(52) \sim(54)$, there exists a $t_{2}=t_{2}\left\{\alpha, \delta, \beta,\left\|f_{0 t}\right\|_{L_{b}^{2}\left(R ; H^{\alpha}\right)}\right.$, $\left.\left\|Y_{0}\right\|_{L_{b}^{2}\left(R ; \Sigma_{0}\right)},\left\|W_{\tau}\right\|_{H^{\alpha} \times H}\right\} \geqslant t_{0}$ such that

$$
E(t) \leqslant \frac{2 C_{0}}{\gamma}
$$

for any $t \geqslant t_{2}$.
By (48),(53), and (55), we get

$$
\begin{aligned}
\left\|(-\Delta)^{\alpha / 2} u\right\|^{2}+\|n\|^{2} & \\
\leqslant & \rho\|n\|^{2}+\rho\left\|(-\Delta)^{\alpha / 2} u\right\|^{2} \\
& +C\left(\rho,\left\|f_{0 t}\right\|_{L_{b}^{2}\left(R ; H^{\alpha}\right)},\left\|Y_{0}\right\|_{L_{b}^{2}\left(R ; \Sigma_{0}\right)}\right) .
\end{aligned}
$$

Then setting $\rho=\min \{\delta / 4, \beta / 2,1 / 2\}$, we get

$$
\begin{aligned}
& \left\|(-\Delta)^{\alpha / 2} u\right\|^{2}+\|n\|^{2} \\
& \quad \leq C\left(\alpha, \delta, \beta,\left\|f_{0 t}\right\|_{L_{b}^{2}\left(R ; H^{\alpha}\right)},\left\|Y_{0}\right\|_{L_{b}^{2}\left(R ; \Sigma_{0}\right)}\right), \quad \forall t \geqslant t_{2} .
\end{aligned}
$$

By using Lemma 14, we conclude the lemma.

Lemma 16. Assume that

(i) $\alpha \geqslant 1$, and $Y(x, t)$ satisfy Assumption 10;

(ii) $W(\tau) \in E_{0}$ and $W(t)$ solves problem (1)-(4).

Then there exist positive constants $C_{3}=C_{3}\left(\alpha, \delta, \beta,\left\|Y_{0}\right\|_{L_{b}^{2}\left(R ; \Sigma_{0}\right)}\right.$, $\left.\left\|f_{0 t}\right\|_{L_{b}^{2}\left(R ; H^{\alpha}\right)}\right)$ and $t_{3}=t_{3}\left(\alpha, \delta, \beta,\left\|Y_{0}\right\|_{L_{b}^{2}\left(R ; \Sigma_{0}\right)},\left\|f_{0 t}\right\|_{L_{b}^{2}\left(R ; H^{\alpha}\right)}\right.$, $\left.\left\|W_{\tau}\right\|_{E_{0}}\right)$ such that

$$
\|W(t)\|_{E_{0}} \leqslant C, \quad \forall t \geqslant t_{3} .
$$

Proof. Taking the inner product of (1) with $(-\Delta)^{\alpha} u_{t}$, we have

$$
\left(i u_{t}-(-\Delta)^{\alpha} u-n u+i \delta u,(-\Delta)^{\alpha} u_{t}\right)=\left(f(x, t),(-\Delta)^{\alpha} u_{t}\right)
$$

Taking the real part of (59), we have

$$
\begin{aligned}
\frac{1}{2} \frac{d}{d t}\left\|(-\Delta)^{\alpha} u\right\|^{2} \\
\quad+\operatorname{Re} \int n u(-\Delta)^{\alpha} \bar{u}_{t} d x+\operatorname{Re} \int i \delta(-\Delta)^{\alpha} \bar{u} u_{t} d x \\
=-\operatorname{Re}\left(f(x, t),(-\Delta)^{\alpha} u_{t}\right),
\end{aligned}
$$


where, by (1) and (2), we have

$$
\begin{aligned}
& \operatorname{Re} \int n u(-\Delta)^{\alpha} \bar{u}_{t} d x \\
& =\frac{d}{d t} \operatorname{Re} \int n u(-\Delta)^{\alpha} \bar{u} d x-\operatorname{Re} \int n_{t} u(-\Delta)^{\alpha} \bar{u} d x \\
& -\operatorname{Re} \int n u_{t}(-\Delta)^{\alpha} \bar{u} d x \\
& =\frac{d}{d t} \operatorname{Re} \int n u(-\Delta)^{\alpha} \bar{u} d x \\
& -\operatorname{Re} \int\left(g(x, t)-\beta n-|u|_{x}^{2}\right) u(-\Delta)^{\alpha} \bar{u} d x \\
& -\operatorname{Re} \int n\left(-i f(x, t)-i(-\Delta)^{\alpha} u-i n u-\delta u\right)(-\Delta)^{\alpha} \bar{u} d x \\
& =\frac{d}{d t} \operatorname{Re} \int n u(-\Delta)^{\alpha} \bar{u} d x \\
& +\operatorname{Re} \int u(-\Delta)^{\alpha} \bar{u}\left(\beta n+|u|_{x}^{2}-g(x, t)\right) d x \\
& +\operatorname{Re} \int n(-\Delta)^{\alpha} \bar{u}\left(i f(x, t)+i(-\Delta)^{\alpha} u+i n u+\delta u\right) d x \\
& =\frac{d}{d t} \operatorname{Re} \int n u(-\Delta)^{\alpha} \bar{u} d x+\operatorname{Re} \int \beta u n(-\Delta)^{\alpha} \bar{u} d x \\
& +\operatorname{Re} \int u^{2} \bar{u}_{x}(-\Delta)^{\alpha} \bar{u} d x+\operatorname{Re} \int|u|^{2} u_{x}(-\Delta)^{\alpha} \bar{u} d x \\
& -\operatorname{Re} \int u g(x, t)(-\Delta)^{\alpha} \bar{u} d x+\operatorname{Re} \int \inf (x, t)(-\Delta)^{\alpha} \bar{u} d x \\
& +\operatorname{Re} \int i n^{2} u(-\Delta)^{\alpha} \bar{u} d x+\operatorname{Re} \int \delta n u(-\Delta)^{\alpha} \bar{u} d x, \\
& \operatorname{Re} \int i \delta(-\Delta)^{\alpha} \bar{u} u_{t} d x \\
& =\operatorname{Re} \int \delta(-\Delta)^{\alpha} \bar{u}\left(f(x, t)+(-\Delta)^{\alpha} u+n u-i \delta u\right) d x \\
& =\delta\left\|(-\Delta)^{\alpha} u\right\|^{2}+\delta \operatorname{Re} \int f(x, t)(-\Delta)^{\alpha} \bar{u} d x \\
& +\delta \operatorname{Re} \int n u(-\Delta)^{\alpha} u d x \\
& \operatorname{Re} \int f(x, t)(-\Delta)^{\alpha} \bar{u}_{t} d x \\
& =\frac{d}{d t} \operatorname{Re} \int f(x, t)(-\Delta)^{\alpha} \bar{u} d x-\operatorname{Re} \int f_{t}(x, t)(-\Delta)^{\alpha} \bar{u} d x .
\end{aligned}
$$

By Lemmas 12,14 , and 15 and $\alpha \geqslant 1$, we see that

$$
\|u\|_{L^{\infty}} \leqslant C\left\|(-\Delta)^{\alpha / 2} u\right\|^{1 / 2 \alpha}\|u\|^{1-(1 / 2 \alpha)}<\infty
$$

By (60) (62), Lemmas 12 15, and Hölder inequality, we can see that

$$
\begin{aligned}
& \frac{d}{d t}\left(\left\|(-\Delta)^{\alpha} u\right\|^{2}+2 \operatorname{Re} \int n u(-\Delta)^{\alpha} \bar{u} d x\right. \\
& \left.+2 \operatorname{Re} \int f(x, t)(-\Delta)^{\alpha} \bar{u} d x\right)+\delta\left\|(-\Delta)^{\alpha} u\right\|^{2} \\
& +\delta\left(\left\|(-\Delta)^{\alpha} u\right\|^{2}+2 \operatorname{Re} \int n u(-\Delta)^{\alpha} \bar{u} d x\right. \\
& \left.+2 \operatorname{Re} \int f(x, t)(-\Delta)^{\alpha} \bar{u} d x\right) \\
& =2 \operatorname{Re} \int u g(x, t)(-\Delta)^{\alpha} \bar{u} d x-2 \operatorname{Re} \int \beta u n(-\Delta)^{\alpha} \bar{u} d x \\
& -2 \operatorname{Re} \int u^{2} \bar{u}_{x}(-\Delta)^{\alpha} \bar{u} d x \\
& -2 \operatorname{Re} \int|u|^{2} u_{x}(-\Delta)^{\alpha} \bar{u} d x-2 \operatorname{Re} \int \inf (x, t)(-\Delta)^{\alpha} \bar{u} d x \\
& -2 \operatorname{Re} \int i n^{2} u(-\Delta)^{\alpha} \bar{u} d x \\
& -2 \operatorname{Re} \int \delta n u(-\Delta)^{\alpha} \bar{u} d x+2 \operatorname{Re} \int f_{t}(x, t)(-\Delta)^{\alpha} \bar{u} d x \\
& \leqslant 2\|u\|_{L^{\infty}}\|g(x, t)\|\left\|(-\Delta)^{\alpha} u\right\|+2 \beta\|u\|_{L^{\infty}}\|n\|\left\|(-\Delta)^{\alpha} u\right\| \\
& +2\|u\|_{L^{\infty}}^{2}\left\|\bar{u}_{x}\right\|\left\|(-\Delta)^{\alpha} u\right\| \\
& +2\|u\|_{L^{\infty}}^{2}\left\|u_{x}\right\|\left\|(-\Delta)^{\alpha} u\right\|+2\|n\|_{L^{4}}\|f(x, t)\|_{L^{4}}\left\|(-\Delta)^{\alpha} u\right\| \\
& +2\|u\|_{L^{\infty}}\|n\|_{L^{4}}^{2}\left\|(-\Delta)^{\alpha} u\right\| \\
& +2 \delta\|u\|_{L^{\infty}}\|n\|\left\|(-\Delta)^{\alpha} u\right\|+2\left\|f_{t}(x, t)\right\|\left\|(-\Delta)^{\alpha} u\right\| \\
& \leqslant\left(2\|u\|_{L^{\infty}}\|g(x, t)\|+2 \beta\|u\|_{L^{\infty}}\|n\|+2 \delta\|u\|_{L^{\infty}}\|n\|\right. \\
& \left.+2\left\|f_{t}(x, t)\right\|\right)\left\|(-\Delta)^{\alpha} u\right\| \\
& +4 C\|u\|_{L^{\infty}}^{2}\left\|(-\Delta)^{\alpha / 2} u\right\|^{1 / \alpha}\|u\|^{1-(1 / \alpha)}\left\|(-\Delta)^{\alpha} u\right\| \\
& +2 C\left\|n_{x}\right\|^{1 / 4}\|n\|^{3 / 4}\left\|(-\Delta)^{\alpha} u\right\| \\
& +2 C\left\|n_{x}\right\|^{1 / 2}\|n\|^{3 / 2}\left\|(-\Delta)^{\alpha} u\right\| \\
& \leqslant C\left\|(-\Delta)^{\alpha} u\right\|+\frac{\beta}{4}\left\|n_{x}\right\|^{2}+C\left\|(-\Delta)^{\alpha} u\right\|^{8 / 7}+\frac{\beta}{4}\left\|n_{x}\right\|^{2} \\
& +C\left\|(-\Delta)^{\alpha} u\right\|^{4 / 3} \\
& \leqslant \frac{\delta}{2}\left\|(-\Delta)^{\alpha} u\right\|+\frac{\beta}{2}\left\|n_{x}\right\|^{2} \\
& +C\left(\alpha, \delta, \beta,\left\|Y_{0}\right\|_{L_{b}^{2}\left(R ; \Sigma_{0}\right)},\left\|f_{0 t}\right\|_{L_{b}^{2}\left(R ; H^{\alpha}\right)}\right) .
\end{aligned}
$$


Taking the inner product of (2) with $n_{x x}$, we have

$$
-\frac{1}{2} \frac{d}{d t}\left\|n_{x}\right\|^{2}+\int|u|_{x}^{2} n_{x x} d x-\beta\left\|n_{x}\right\|^{2}=\int g(x, t) n_{x x} d x
$$

where, by Lemmas $12 \sim 15$ and $\alpha \geqslant 1$, we can see that

$$
\begin{aligned}
& \left.\left|\int\right| u\right|_{x} ^{2} n_{x x} d x \mid \\
& =\left|\int\left(u_{x} \bar{u}+u \bar{u}_{x}\right) n_{x x} d x\right| \\
& =\left.\left|-2 \operatorname{Re} \int u \bar{u}_{x x} n_{x} d x-2 \int\right| u_{x}\right|^{2} n_{x} d x \mid \\
& \leqslant\left|-2 \operatorname{Re} \int u \bar{u}_{x x} n_{x} d x\right| \\
& +\left|2 \int\left(u_{x x} \bar{u}_{x}+u_{x} \bar{u}_{x x}\right) n d x\right| \\
& \leqslant 2\|u\|_{L^{\infty}}\left\|\bar{u}_{x x}\right\|\left\|n_{x}\right\|+4\left\|u_{x}\right\|_{L^{4}}\left\|u_{x x}\right\|\|n\|_{L^{4}} \\
& \leqslant C\left\|(-\Delta)^{\alpha} u\right\|^{1 / \alpha}\|u\|^{1-(1 / \alpha)}\left\|n_{x}\right\| \\
& +C\left\|(-\Delta)^{\alpha} u\right\|^{5 / 8 \alpha}\|u\|^{1-(5 / 8 \alpha)}\left\|(-\Delta)^{\alpha} u\right\|^{1 / \alpha} \\
& \times\|u\|^{1-(1 / \alpha)}\left\|n_{x}\right\|^{1 / 4}\|n\|^{3 / 4} \\
& \leqslant \frac{\delta}{16}\left\|(-\Delta)^{\alpha} u\right\|^{2}+C\left\|n_{x}\right\|^{2 \alpha /(2 \alpha-1)} \\
& +\frac{\beta}{16}\left\|n_{x}\right\|^{2}+C\left\|(-\Delta)^{\alpha} u\right\|^{13 / 7 \alpha} \\
& \leqslant \frac{\delta}{4}\left\|(-\Delta)^{\alpha} u\right\|^{2}+\frac{\beta}{8}\left\|n_{x}\right\|^{2} \\
& +C\left(\alpha, \delta, \beta,\left\|Y_{0}\right\|_{L_{b}^{2}\left(R ; \Sigma_{0}\right)}\right)
\end{aligned}
$$

So by (64) and (65), we get

$$
\begin{aligned}
& \frac{d}{d t}\left\|n_{x}\right\|^{2}+2 \beta\left\|n_{x}\right\|^{2} \\
&=-2 \int g(x, t) n_{x x} d x+2 \int|u|_{x}^{2} n_{x x} d x \\
&=2 \int g_{x}(x, t) n_{x} d x+2 \int|u|_{x}^{2} n_{x x} d x \\
& \leqslant 2\left\|g_{x}(x, t)\right\|\left\|n_{x}\right\|+\frac{\delta}{2}\left\|(-\Delta)^{\alpha} u\right\|^{2} \\
&+\frac{\beta}{4}\left\|n_{x}\right\|^{2}+C\left(\alpha, \delta, \beta,\left\|Y_{0}\right\|_{L_{b}^{2}\left(R ; \Sigma_{0}\right)}\right) \\
& \leqslant \frac{\delta}{2}\left\|(-\Delta)^{\alpha} u\right\|^{2}+\frac{\beta}{2}\left\|n_{x}\right\|^{2} \\
&+C\left(\alpha, \delta, \beta,\left\|Y_{0}\right\|_{L_{b}^{2}\left(R ; \Sigma_{0}\right)}\right) .
\end{aligned}
$$

Set $\gamma=\min \{\delta, \beta\}$ and

$$
\begin{aligned}
E= & \left\|(-\Delta)^{\alpha} u\right\|^{2}+\left\|n_{x}\right\|^{2}+2 \operatorname{Re} \int n u(-\Delta)^{\alpha} \bar{u} d x \\
& +2 \operatorname{Re} \int f(x, t)(-\Delta)^{\alpha} \bar{u} d x .
\end{aligned}
$$

Then by (63) and (66), we can deduce that

$$
\frac{d}{d t} E+\gamma E \leqslant C\left(\alpha, \delta, \beta,\left\|f_{0 t}\right\|_{L_{b}^{2}\left(R ; H^{\alpha}\right)},\left\|Y_{0}\right\|_{L_{b}^{2}\left(R ; \Sigma_{0}\right)}\right) \quad \forall t \geqslant t_{3^{*}},
$$

which has the same form with (51) in the proof of Lemma 15. Similar to the study of (51), there exist positive constants $C_{3}=C_{3}\left(\alpha, \delta, \beta,\left\|Y_{0}\right\|_{L_{b}^{2}\left(R ; \Sigma_{0}\right)},\left\|f_{0 t}\right\|_{L_{b}^{2}\left(R ; H^{\alpha}\right)}\right)$ and $t_{3}=$ $t_{3}\left(\alpha, \delta, \beta,\left\|W_{\tau}\right\|_{E_{0}},\left\|f_{0 t}\right\|_{L_{b}^{2}\left(R ; H^{\alpha}\right)},\left\|Y_{0}\right\|_{L_{b}^{2}\left(R ; \Sigma_{0}\right)}\right)$ such that

$$
\left\|(-\Delta)^{\alpha} u\right\|^{2}+\left\|n_{x}\right\|^{2} \leqslant \frac{1}{2}\left(\left\|(-\Delta)^{\alpha} u\right\|^{2}+\left\|n_{x}\right\|^{2}\right)+C_{3},
$$

which conclude the proof of Lemma 16.

\section{Unique Existence of the Solution}

In this section, we show the unique existence theorem of the solutions. Since uniform a priori estimates have been established in the above section, one can readily get the existence of the solution by Galërkin's method (see [20, 22$24]$ ). We show the theorem and prove it briefly for readers' convenience.

Theorem 17. Set $\alpha \geqslant 1$, and $Y(x, t)$ satisfy Assumption 10; for each $W_{\tau} \in E_{0}$, then system (1)-(4) has a unique global solution $W(x, t) \in L^{\infty}\left(\tau, T ; E_{0}\right), \forall T>\tau$.

Proof. We prove this theorem by two steps.

Step 1. The existence of solution.

By Galërkin's method, we construct the approximate solution of the periodic initial value problem (1) (4). We apply the following approximate solution:

$$
\begin{array}{ll}
u^{l}(x, t)=\sum_{j=1}^{l} a_{j}^{l}(t) \eta_{j}(x), & n^{l}(x, t)=\sum_{j=1}^{l} b_{j}^{l}(t) \eta_{j}(x), \\
& \eta_{j}(x)=e^{i j x}, \\
W^{l}(x, t)=\left(u^{l}(x, t), n^{l}(x, t)\right), &
\end{array}
$$

to approach $W(x, t)$, the solution of the problem (1)-(4). And for $j=1,2, \ldots, l, W^{l}(x, t)$ satisfies

$$
\begin{gathered}
\left(i u_{t}^{l}-(-\Delta)^{\alpha} u^{l}-n^{l} u^{l}+i \delta u^{l}-f(x, t), \eta_{j}\right)=0, \\
\left(n_{t}^{l}+\beta n^{l}+\left|u^{l}\right|_{x}^{2}-g(x, t), \eta_{j}\right)=0, \\
\left(W^{l}(x, \tau), \eta_{j}\right)=\left(W_{\tau}, \eta_{j}\right), \\
W^{l}(x+2 \pi, t)=W^{l}(x, t) .
\end{gathered}
$$


We see that system (71) is an initial boundary value problem of ordinary differential equations (ODE). By the standard existence theory for ODE and uniform a priori estimates in Section 3, for any $l$, there exists a unique solution of (71), such that

$$
\begin{aligned}
& \sup _{\tau \leqslant t \leqslant \infty}\left(\left\|u^{l}\right\|_{H^{\alpha}}+\left\|n^{l}\right\|_{H^{1}}+\left\|u_{t}^{l}\right\|_{L^{2}}+\left\|n_{t}^{l}\right\|_{H^{1}}\right) \\
& \leqslant C\left(\alpha, \delta, \beta,\left\|Y_{0}\right\|_{L_{b}^{2}\left(R ; \Sigma_{0}\right)},\left\|f_{0 t}\right\|_{L_{b}^{2}\left(R ; H^{\alpha}\right)},\left\|W_{\tau}\right\|_{E_{0}}\right) .
\end{aligned}
$$

There is a subsequence $W^{l_{k}}(x, t)$ of $W^{l}(x, t)$ and $W(x, t)=$ $(u(x, t), n(x, t))$ such that

$$
\begin{gathered}
W^{l_{k}}(x, t) \longrightarrow W(x, t), \quad * \text {-weakly in } L^{\infty}\left(\tau, T ; E_{0}\right), \\
W_{t}^{l_{k}}(x, t) \longrightarrow W(x, t),
\end{gathered}
$$$$
\text { *-weakly in } L^{\infty}\left(\tau, T ; L^{2}(\Omega) \times H^{1}(\Omega)\right) \text {. }
$$

Due to the above proof and the continuous extension theorem, $W(x, t)$ is the solution of (1) (4).

Step 2. The uniqueness of solution.

Suppose $W_{1}(x, t)=\left(u_{1}(x, t), n_{1}(x, t)\right), W_{2}(x, t)=$ $\left(u_{2}(x, t), n_{2}(x, t)\right)$ are two solutions of problem $(1)-(4)$. Let $W(x, t)=W_{1}(x, t)-W_{2}(x, t)$, and then $W(x, t)=$ $(u(x, t), n(x, t))$ satisfies

$$
\begin{gathered}
i u_{t}-(-\Delta)^{\alpha} u-n_{1} u_{1}+n_{2} u_{2}+i \delta u=0, \\
n_{t}+\beta n+\left|u_{1}\right|_{x}^{2}-\left|u_{2}\right|_{x}^{2}=0, \\
W(x, \tau)=0 \\
W(x+2 \pi, t)=W(x, t) .
\end{gathered}
$$

Obviously, $\|W(x, t)\|_{E_{0}}$ is uniformly bounded. Note that $-n_{1} u_{1}+n_{2} u_{2}=-n u_{1}-n_{2} u,\left|u_{1}\right|_{x}^{2}-\left|u_{2}\right|_{x}^{2}=u_{x} \bar{u}_{1}+u_{2 x} \bar{u}+$ $u \bar{u}_{1 x}+u_{2} \bar{u}_{x}$.

Taking the inner product of (74) with $u$ and taking the imaginary part, we can get

$$
\begin{aligned}
\frac{d}{d t}\|u\|^{2} & =-2 \delta\|u\|^{2}+2 \operatorname{Im}\left(n u_{1}+n_{2} u, u\right) \\
& \leqslant 2 \delta\|u\|^{2}+2\left\|u_{1}\right\|_{L^{\infty}}\|n\|\|u\| \\
& \leqslant C\left(\|u\|^{2}+\|n\|^{2}\right) .
\end{aligned}
$$

Taking the inner product of (75) with $n$, we can obtain

$$
\begin{aligned}
\frac{d}{d t}\|n\|^{2}= & -2 \beta\|n\|^{2}-2\left(u_{x} \bar{u}_{1}+u_{2 x} \bar{u}+u \bar{u}_{1 x}+u_{2} \bar{u}_{x}, n\right) \\
\leqslant & 2 \beta\|n\|^{2}+2\left(\left\|u_{x}\right\|\left\|u_{1}\right\|_{L^{\infty}}+\left\|u_{2 x}\right\|_{L^{\infty}}\|u\|\right. \\
& \left.\quad+\|u\|\left\|u_{1 x}\right\|_{L^{\infty}}+\left\|u_{2}\right\|_{L^{\infty}}\left\|u_{x}\right\|\right)\|n\| \\
\leqslant & 2 \beta\|n\|^{2}+C\left(\left\|(-\Delta)^{\alpha / 2} u\right\|^{1 / \alpha}\|u\|^{1-(1 / \alpha)}+\|u\|\right)\|n\| \\
\leqslant & 2 \beta\|n\|^{2}+C\left(\left\|(-\Delta)^{\alpha / 2} u\right\|+\|u\|\right)\|n\| \\
\leqslant & C\left(\|u\|^{2}+\|n\|^{2}+\left\|(-\Delta)^{\alpha / 2} u\right\|^{2}\right)
\end{aligned}
$$

Taking the inner product of (74) with $u_{t}$ and taking the real part, we can get

$$
\begin{aligned}
\frac{d}{d t} \|( & -\Delta)^{\alpha / 2} u \|^{2} \\
& =2 \operatorname{Re}\left(i \delta u-n u_{1}-n_{2} u, u_{t}\right) \\
& \leqslant C\left(\|u\|+\left\|u_{1}\right\|_{L^{\infty}}\|n\|+\left\|n_{2}\right\|_{L^{\infty}}\|u\|\right)\left\|u_{t}\right\| \\
& \leqslant C\left(\|u\|^{2}+\|n\|^{2}+\left\|u_{t}\right\|^{2}\right) .
\end{aligned}
$$

Differentiating (74) with respect to $t$, taking the inner product of with $u_{t}$, and taking the imaginary part, we can get

$$
\begin{aligned}
\frac{d}{d t}\left\|u_{t}\right\|^{2} & \\
= & \operatorname{Im}\left(n_{t} u_{1}, u_{t}\right)+2 \operatorname{Im}\left(n u_{1 t}, u_{t}\right)+2 \operatorname{Im}\left(n_{2 t} u, u_{t}\right) \\
\leqslant & C\left(\left\|u_{1}\right\|_{L^{\infty}}\left\|n_{t}\right\|+\left\|u_{1 t}\right\|\|n\|_{L^{\infty}}\right. \\
& \left.\quad+\left\|n_{2 t}\right\|_{L^{\infty}}\|u\|\right)\left\|u_{t}\right\| \\
\leqslant & C\left\|-\beta n-u_{x} \bar{u}_{1}-u_{2 x} \bar{u}-u \bar{u}_{1 x}-u_{2} \bar{u}_{x}\right\|\left\|u_{t}\right\| \\
& +C\left(\left\|n_{x}\right\|^{1 / 2}\|n\|^{1 / 2}+\|u\|\right)\left\|u_{t}\right\| \\
\leqslant & C\left(\|n\|+\left\|n_{x}\right\|+\left\|u_{x}\right\|+\|u\|\right)\left\|u_{t}\right\| \\
\leqslant & C\left(\|n\|+\left\|n_{x}\right\|+\left\|(-\Delta)^{\alpha / 2} u\right\|^{1 / \alpha}\|u\|^{1-(1 / \alpha)}+\|u\|\right)\left\|u_{t}\right\| \\
\leqslant & C\left(\|n\|^{2}+\left\|n_{x}\right\|^{2}+\left\|(-\Delta)^{\alpha / 2} u\right\|^{2}+\|u\|^{2}+\left\|u_{t}\right\|^{2}\right) .
\end{aligned}
$$

Taking the inner product of (75) with $n_{x x}$, we have

$$
\begin{aligned}
& \frac{d}{d t}\left\|n_{x}\right\|^{2} \\
& \quad=-2 \beta\left\|n_{x}\right\|^{2}+2\left(u_{x} \bar{u}_{1}+u_{2 x} \bar{u}+u \bar{u}_{1 x}+u_{2} \bar{u}_{x}, n_{x x}\right)
\end{aligned}
$$




$$
\begin{aligned}
= & -2 \beta\left\|n_{x}\right\|^{2}-2 \int\left(u_{x x} \bar{u}_{1}+u_{x} \bar{u}_{1 x}\right) n_{x} d x \\
& -2 \int\left(u_{2 x x} \bar{u}+u_{2 x} \bar{u}_{x}\right) n_{x} d x \\
& -2 \int\left(u_{x} \bar{u}_{1 x}+u \bar{u}_{1 x x}\right) n_{x} d x \\
& -2 \int\left(u_{2 x} \bar{u}_{x}+u_{2} \bar{u}_{x x}\right) n_{x} d x \\
\leqslant & 2 \beta\left\|n_{x}\right\|^{2}+C\left(\|u\|+\left\|u_{x}\right\|+\left\|u_{x x}\right\|\right)\left\|n_{x}\right\| \\
\leqslant & 2 \beta\left\|n_{x}\right\|^{2}+C\left(\|u\|+\left\|(-\Delta)^{\alpha / 2} u\right\|^{1 / \alpha}\|u\|^{1-(1 / \alpha)}\right. \\
& \left.+\left\|(-\Delta)^{\alpha} u\right\|^{1 / \alpha}\|u\|^{1-(1 / \alpha)}\right)\left\|n_{x}\right\|^{2} \\
\leqslant & C\left(\|u\|^{2}+\left\|(-\Delta)^{\alpha / 2} u\right\|^{2}+\left\|(-\Delta)^{\alpha} u\right\|^{2}+\left\|n_{x}\right\|^{2}\right) \\
\leqslant & C\left(\|u\|^{2}+\left\|(-\Delta)^{\alpha / 2} u\right\|^{2}\right. \\
& \left.+\left\|i u_{t}-n u_{1}-n_{2} u+i \delta u\right\|^{2}+\left\|n_{x}\right\|^{2}\right) \\
\leqslant & C\left(\|u\|^{2}+\|n\|^{2}+\left\|(-\Delta)^{\alpha / 2} u\right\|^{2}+\left\|u_{t}\right\|^{2}+\left\|n_{x}\right\|^{2}\right) .
\end{aligned}
$$

Therefore by (78) (81), we conclude that

$$
\begin{aligned}
& \frac{d}{d t}\left(\|u\|^{2}+\|n\|^{2}+\left\|(-\Delta)^{\alpha / 2} u\right\|^{2}+\left\|n_{x}\right\|^{2}+\left\|u_{t}\right\|^{2}\right) \\
& \leqslant C\left(\|u\|^{2}+\|n\|^{2}+\left\|(-\Delta)^{\alpha / 2} u\right\|^{2}+\left\|n_{x}\right\|^{2}+\left\|u_{t}\right\|^{2}\right) .
\end{aligned}
$$

From Gronwall inequality and (76), we have

$$
u(x, t)=0, \quad n(x, t)=0 .
$$

Therefore, we complete the proof of the theorem.

\section{Uniform Absorbing Set and Uniform Attractor}

In this section, we will prove the existence of the strong compact uniform attractor of problem (1) (4) applying Ball et al.s idea (see $[19,22])$. Firstly, we construct a bounded uniformly absorbing set. Next, we show the weak uniform attractor of the system. Lastly, we derive that the weak uniform attractor is actually the strong one.

Theorem 18. Under assumptions of Theorem 17, $\left\{U_{\sigma \in \Sigma}(t, \tau)\right\}$ admits a strong compact uniform attractor $\mathscr{A}_{\sum}$.

Proof. We prove this theorem by three steps.

Step 1. $\left\{U_{\sigma \in \Sigma}(t, \tau)\right\}$ possess a bounded uniformly absorbing set in $E_{0}$.

Let $B_{0}=\left\{W \quad \in E_{0}\|W\|_{E_{0}}^{2} \leqslant C\left(\alpha, \delta, \beta,\left\|Y_{0}\right\|_{L_{c}^{2}\left(\mathbb{R} ; \Sigma_{0}\right)}\right.\right.$, $\left.\left.\left\|f_{0 t}\right\|_{L^{2}\left(R ; H^{\alpha}\right)},\left\|W_{\tau}\right\|_{E_{0}}\right)\right\}$. By Theorem 17, $B_{0}$ is a bounded absorbing set of the process $U_{\sigma=Y_{0}}$.
By Assumption 10, we know that, for each $Y \in \sum$, $\|Y\|_{L_{b}^{2}\left(\mathbb{R} ; \Sigma_{0}\right)}^{2} \leqslant\left\|Y_{0}\right\|_{L_{b}^{2}\left(\mathbb{R} ; \Sigma_{0}\right)}^{2}$ holds. So the solution of $(1) \sim(4)$ satisfies

$$
\begin{aligned}
\|W\|_{E_{0}} & \leqslant C\left(\alpha, \delta, \beta,\left\|f_{0 t}\right\|_{L^{2}\left(R ; H^{\alpha}\right)},\|Y\|_{L_{b}^{2}\left(\mathbb{R} ; \sum_{0}\right)},\left\|W_{\tau}\right\|_{E_{0}}\right) \\
& \leqslant C\left(\alpha, \delta, \beta,\left\|f_{0 t}\right\|_{L^{2}\left(R ; H^{\alpha}\right)},\left\|Y_{0}\right\|_{L_{b}^{2}\left(\mathbb{R} ; \sum_{0}\right),\left\|W_{\tau}\right\|_{E_{0}}}\right) .
\end{aligned}
$$

Then we can get that the set $B_{0}$ is a bounded uniformly absorbing set of $\left\{U_{\sigma \in \Sigma}(t, \tau)\right\}$.

Step 2. we prove the existence of weakly compact uniform attractor $\mathscr{A}_{\Sigma}$ in $E_{0}$.

From Lemma 6, Theorem 17, and Step 1, we only need to prove that $\left\{U_{\sigma \in \Sigma}(t, \tau)\right\}$ is $\left(E_{0} \times \sum, E_{0}\right)$-continuous. We denote weak convergence by $\rightarrow$ and $*$ weak convergence by $\stackrel{*}{\rightarrow}$.

For any fixed $t_{1} \geqslant \tau \in \mathbb{R}$, let

$$
\left(W_{\tau k}, \sigma_{k}\right) \rightarrow\left(W_{\tau}, \sigma\right) \text { in } E_{0} \times \sum \text {. }
$$

If we can deduce that

$$
W_{\sigma_{k}}\left(t_{1}\right) \rightarrow W_{\sigma}\left(t_{1}\right) \text { in } E_{0},
$$

where $W_{\sigma_{k}}\left(t_{1}\right)=\left(u_{k}\left(t_{1}\right), n_{k}\left(t_{1}\right)\right)=U_{\sigma_{k}}\left(t_{1}, \tau\right) W_{\tau k}, W_{\sigma}\left(t_{1}\right)=$ $\left(u\left(t_{1}\right), n\left(t_{1}\right)\right)=U_{\sigma}\left(t_{1}, \tau\right) W_{\tau}$, we will obtain that $\left\{U_{\sigma \in \Sigma}(t, \tau)\right\}$ is $\left(E_{0} \times \sum, E_{0}\right)$-continuous. By (86) and Theorem 17, we can get that

$$
\begin{gathered}
\left\|W_{\tau k}\right\|_{E_{0}} \leqslant C, \\
\sup _{t \in[\tau, T]}\left\|W_{\sigma_{k}}(t)\right\|_{E_{0}} \leqslant C .
\end{gathered}
$$

Then by Lemmas 12 16, we can see that

$$
\left\|W_{\sigma_{k}}(t)\right\|_{\infty} \leqslant C, \quad \forall 0 \leqslant t \leqslant T .
$$

Note that

$$
\begin{aligned}
i u_{k t} & =(-\Delta)^{\alpha} u_{k}+n_{k} u_{k}-i \delta u_{k}+f_{k}(x, t), \\
n_{k t} & =-\left|u_{k}\right|_{x}^{2}-\beta n_{k}+g_{k}(x, t)
\end{aligned}
$$

and $\sigma_{k}=\left(f_{k}(x, t), g_{k}(x, t)\right) \in \sum$. By (89) and (90), we find that $\partial_{t} W_{\sigma_{k}}(t) \in L^{\infty}\left(\tau, T ; L^{2}(\Omega) \times H^{1}(\Omega)\right)$ and

$$
\left\|\partial_{t} W_{\sigma_{k}}(t)\right\|_{L^{\infty}\left(\tau, T ; L^{2}(\Omega) \times H^{1}(\Omega)\right)} \leqslant C .
$$

Because of Theorem 17 and (93), we easily see that there exist a subsequence $\left\{W_{\sigma_{k_{l}}}(t)\right\}$ of $\left\{W_{\sigma_{k}}(t)\right\}$ and $\widetilde{W}(t) \triangleq(\widetilde{u}(t), \widetilde{n}(t)) \epsilon$ $L^{\infty}\left(\tau, T ; E_{0}\right)$, such that

$$
\begin{gathered}
W_{\sigma_{k_{l}}}(t) \stackrel{*}{\rightarrow} \widetilde{W}(t) \quad \text { in } L^{\infty}\left(\tau, T ; E_{0}\right), \\
\partial_{t} W_{\sigma_{k_{l}}}(t) \stackrel{*}{\rightarrow} \partial_{t} \widetilde{W}(t) \quad \text { in } L^{\infty}\left(\tau, T ; L^{2}(\Omega) \times H^{1}(\Omega)\right) .
\end{gathered}
$$

Besides, for any $t_{1} \in[\tau, T]$, by (89) there exists $W^{0} \triangleq$ $\left(u^{0}\left(t_{1}\right), n^{0}\left(t_{1}\right)\right) \in E_{0}$ such that

$$
W_{\sigma_{k}}\left(t_{1}\right) \rightarrow W^{0} \text { in } E_{0} .
$$


By (94) and compactness embedding theorem, we can get that

$$
u_{k_{l}}(t) \longrightarrow \widetilde{u}(t) \quad \text { in } L^{2}\left(\tau, T ; H^{\alpha}\right) .
$$

Next, we will obtain that $\widetilde{W}(t)$ is a solution of problem (1) (4).

For all $v \in L^{2}(\Omega), \forall \psi \in C_{0}^{\infty}(\tau, T)$, by (91) we have that

$$
\begin{aligned}
& \int_{\tau}^{T}\left(i u_{k_{l}}, \psi(t) v\right) d t-\int_{\tau}^{T}\left((-\Delta)^{\alpha} u_{k_{l}}, \psi(t) v\right) d t \\
& \quad-\int_{\tau}^{T}\left(n_{k_{l}} u_{k_{l}}, \psi(t) v\right) d t+\int_{\mathcal{\tau}}^{T}\left(i \delta u_{k_{l}}, \psi(t) v\right) d t \\
& \quad-\int_{\tau}^{T}(f(x, t), \psi(t) v) d t=0 .
\end{aligned}
$$

by (90), (94), and (97),

$$
\begin{aligned}
& \int_{\tau}^{T}\left(\left(u_{k_{l}}-\tilde{u}\right) n_{k_{l}}, \psi(t) v\right) d t \\
& \leqslant \sup _{0 \leqslant t \leqslant T}\left\|n_{k_{l}}(t)\right\|_{L^{\infty}}\|\psi(t) v\|_{L^{2}\left(0, T ; L^{2}(\Omega)\right)} \\
& \quad \times\left\|u_{k_{l}}-\tilde{u}\right\|_{L^{2}\left(0, T ; L^{2}(\Omega)\right)} \longrightarrow 0, \\
& \int_{\tau}^{T}\left(\tilde{u}\left(n_{k_{l}}-\tilde{n}\right), \psi(t) v\right) d t \\
& \quad=\int_{\tau}^{T}\left(\left(n_{k_{l}}-\tilde{n}\right), \psi(t) v \overline{\tilde{u}}\right) d t \longrightarrow 0 .
\end{aligned}
$$

Then we have

$$
\int_{\tau}^{T}\left(n_{k_{l}} u_{k_{l}}, \psi(t) v\right) d t \longrightarrow \int_{\tau}^{T}(\widetilde{n} \tilde{\mathcal{u}}, v) \psi(t) d t
$$

And by (94), we have that

$$
\begin{gathered}
\int_{\tau}^{T}\left((-\Delta)^{\alpha} u_{k_{l}}, \psi(t) v\right) d t-\int_{\tau}^{T}\left((-\Delta)^{\alpha} \widetilde{u}, \psi(t) v\right) d t \\
\leqslant\left\|(-\Delta)^{\alpha}\left(u_{k_{l}}-\widetilde{u}\right)\right\|_{L^{2}\left(\tau, T ; L^{2}(\Omega)\right)} \\
\quad \times\|\psi(t) v\|_{L^{2}\left(\tau, T ; L^{2}(\Omega)\right)} \longrightarrow 0 .
\end{gathered}
$$

By using the similar methods to the other terms of (98), we have

$$
\begin{gathered}
\int_{\tau}^{T}\left(i \widetilde{u}_{t}, v\right) \psi(t) d t-\int_{\tau}^{T}\left((-\Delta)^{\alpha} \widetilde{\mathcal{u}}, v\right) \psi(t) d t \\
\quad-\int_{\tau}^{T}(\widetilde{n} \widetilde{\mathcal{u}}, v) \psi(t) d t+\int_{\tau}^{T}(i \delta \widetilde{u}, v) \psi(t) d t \\
-\int_{\tau}^{T}(f(x, t), v) \psi(t) d t=0 .
\end{gathered}
$$

So, we can get that

$$
i \widetilde{u}_{t}-(-\Delta)^{\alpha} \tilde{u}-\tilde{u} \widetilde{n}+i \delta \tilde{u}=f(x, t),
$$

which shows that $(\widetilde{u}, \widetilde{n})$ satisfies (1).

For any $v \in L^{2}(\Omega), \forall \psi \in C_{0}^{\infty}(\tau, T)$ with $\psi(T)=0, \psi(\tau)=$ 1 , by (91) we find that

$$
\begin{gathered}
-\int_{\tau}^{T}\left(i u_{k_{l}}, v\right) \psi^{\prime}(t) d t-\int_{\tau}^{T}\left((-\Delta)^{\alpha} u_{k_{l}}, v\right) \psi(t) d t \\
-\int_{\tau}^{T}\left(n_{k_{l}} u_{k_{l}}, v\right) \psi(t) d t+\int_{\tau}^{T}\left(i \delta u_{k_{l}}, v\right) \psi(t) d t \\
-\int_{\tau}^{T}(f(x, t), v) \psi(t) d t=i\left(u_{k_{l}}(\tau), v\right) .
\end{gathered}
$$

We know that Assumption (86) implies that

$$
u_{k_{l}}(\tau)=u_{\tau k_{l}} \rightarrow u_{\tau} \text { in } H^{2 \alpha} .
$$

Then from (105) and (106), we have

$$
\begin{gathered}
-\int_{\tau}^{T}(i \widetilde{u}, v) \psi^{\prime}(t) d t-\int_{\tau}^{T}\left((-\Delta)^{\alpha} \widetilde{\mathcal{u}}, v\right) \psi(t) d t \\
-\int_{\tau}^{T}(\tilde{n} \tilde{\mathcal{u}}, v) \psi(t) d t+\int_{\tau}^{T}(i \delta \tilde{u}, v) \psi(t) d t \\
-\int_{\tau}^{T}(f(x, t), v) \psi(t) d t=i\left(u_{\tau}, v\right),
\end{gathered}
$$

while by (104) we know that

$$
\begin{array}{r}
-\int_{\tau}^{T}(i \widetilde{u}, v) \psi^{\prime}(t) d t-\int_{\tau}^{T}\left((-\Delta)^{2} \widetilde{u}, v\right) \psi(t) d t \\
-\int_{\tau}^{T}(\widetilde{n} \widetilde{u}, v) \psi(t) d t+\int_{\tau}^{T}(i \delta \widetilde{u}, v) \psi(t) d t \\
-\int_{\tau}^{T}(f(x, t), v) \psi(t) d t=i(\widetilde{u}(\tau), v) .
\end{array}
$$

So by (107) and (108), we have that

$$
\begin{gathered}
\left(u_{\tau}, v\right)=(\widetilde{u}(\tau), v), \quad \forall v \in L^{2}(\Omega), \\
\widetilde{u}(\tau)=u_{\tau} .
\end{gathered}
$$

By (104) and (110), we have

$$
\tilde{u}(t)=u(t) .
$$


For any $v \in L^{2}(\Omega), \forall \psi \in C_{0}^{\infty}\left(\tau, t_{1}\right)$, with $\psi(\tau)=0, \psi\left(t_{1}\right)=1$, then we repeat the procedure of proofs of (105) (108) by (96) having

$$
u^{0}\left(t_{1}\right)=\tilde{u}\left(t_{1}\right)
$$

From (96), (111), and (112), we have that

$$
u_{k}\left(t_{1}\right) \rightarrow u\left(t_{1}\right) \text { in } H^{2 \alpha}(\Omega) .
$$

Similarly, we can also derive that

$$
n_{k}\left(t_{1}\right) \rightarrow n\left(t_{1}\right) \quad \text { in } H^{1}(\Omega) .
$$

From (113) and (114), we deduce (87). We complete the proof of the step.

Step 3. We show the weakly compact uniform attractor $\mathscr{A}_{\Sigma}$ is actually the strong one.

From the proof of Lemma 16, we know each solution trajectory for problem (1)-(4) satisfies

$$
\begin{gathered}
\frac{d}{d t}\left(\left\|u_{x x}\right\|^{2}+F\right)+2 \delta\left(\left\|u_{x x}\right\|^{2}+F\right)=G, \\
\frac{d}{d t}\left\|n_{x}\right\|^{2}+2 \beta\left\|n_{x}\right\|^{2}=G_{1},
\end{gathered}
$$

where

$$
\begin{aligned}
F= & 2 \operatorname{Re} \int n u(-\Delta)^{\alpha} \bar{u} d x+2 \operatorname{Re} \int f(x, t)(-\Delta)^{\alpha} \bar{u} d x \\
G=2 & \operatorname{Re} \int u g(x, t)(-\Delta)^{\alpha} \bar{u} d x-2 \operatorname{Re} \int \beta u n(-\Delta)^{\alpha} \bar{u} d x \\
& -2 \operatorname{Re} \int u^{2} \bar{u}_{x}(-\Delta)^{\alpha} \bar{u} d x-2 \operatorname{Re} \int|u|^{2} u_{x}(-\Delta)^{\alpha} \bar{u} d x \\
& -2 \operatorname{Re} \int i n f(x, t)(-\Delta)^{\alpha} \bar{u} d x-2 \operatorname{Re} \int i n^{2} u(-\Delta)^{\alpha} \bar{u} d x \\
& +2 \operatorname{Re} \int f_{t}(x, t)(-\Delta)^{\alpha} \bar{u} d x \\
& +2 \operatorname{Re} \int \delta f(x, t)(-\Delta)^{\alpha} \bar{u} d x, \\
G_{1}= & -2 \int g(x, t) n_{x x} d x+2 \int|u|_{x}^{2} n_{x x} d x \\
= & 2 \int g_{x}(x, t) n_{x} d x-2 \operatorname{Re} \int u \bar{u}_{x x} n_{x} d x \\
& +2 \int\left(u_{x x} \bar{u}_{x}+u_{x} \bar{u}_{x x}\right) n d x .
\end{aligned}
$$

By the uniform boundedness and the compactness embedding, we have that $F, G$, and $G_{1}$ are all weakly continuous in $E_{0} \times \Sigma$.

From Step 2, we can see that the point $(w, m) \in \mathscr{A}_{\Sigma}$ if and only if there exist two sequences $\left\{w_{k}^{0}, m_{k}^{0}\right\}_{k=1}^{\infty}$ and $\left\{t_{k}\right\}_{k=1}^{\infty}$ such that for all $\sigma(t) \in \Sigma$, it uniformly satisfies that

$$
U_{\sigma}\left(t_{k}, \tau\right)\left(w_{k}^{0}, m_{k}^{0}\right) \rightarrow(w, m) \quad \text { in } E_{0}, k \longrightarrow \infty
$$

where $t_{k} \rightarrow \infty$ as $k \rightarrow \infty$. If the weak convergence implies strong one, we obtain $\mathscr{A}_{\Sigma}$ is actually the strong compact attractor. For each fixed $h>\tau$, because of $t_{k} \rightarrow \infty$, we consider it as $h<t_{k}, k \in N_{+}$. By Lemma 16 and Theorem 17, $U_{\sigma}\left(t_{k}-h, \tau\right)\left(w_{k}^{0}, m_{k}^{0}\right)$ is bounded in $E_{0}$. Then there exists a subsequence $U_{\sigma}\left(t_{k_{l}}-h, \tau\right)\left(w_{k_{l}}^{0}, m_{k_{l}}^{0}\right)$ of $U_{\sigma}\left(t_{k}-h, \tau\right)\left(w_{k}^{0}, m_{k}^{0}\right)$ and a point $(v, p) \in E_{0}$, such that

$$
U_{\sigma}\left(t_{k_{l}}-h, \tau\right)\left(w_{k_{l}}^{0}, m_{k_{l}}^{0}\right) \rightarrow(v, p) \quad \text { in } E_{0}
$$

Let

$$
\begin{aligned}
& \left(w_{k_{l}}(t), m_{k_{l}}(t)\right) \\
& \quad=U_{T\left(t_{k_{l}}-h-\tau\right) \sigma}(t+\tau, \tau) U_{\sigma}\left(t_{k_{l}}-h, \tau\right)\left(w_{k_{l}}^{0}, m_{k_{l}}^{0}\right) \\
& \quad=U_{\sigma}\left(t+t_{k_{l}}-h, t_{k_{l}}-h\right) U_{\sigma}\left(t_{k_{l}}-h, \tau\right)\left(w_{k_{l}}^{0}, m_{k_{l}}^{0}\right) \\
& \quad=U_{\sigma}\left(t+t_{k_{l}}-h, \tau\right)\left(w_{k_{l}}^{0}, m_{k_{l}}^{0}\right)
\end{aligned}
$$

where $T(\cdot)$ is the translation operator on $\Sigma$. Since $\sigma(t)$ is translation compact symbol, there exists a symbol $\sigma^{*} \in \Sigma$ such that

$$
T\left(t_{k_{l}}-h-\tau\right) \sigma \longrightarrow \sigma^{*} \text { in } \Sigma \text {. }
$$

Then by (118), (119), and the weak $(E \times \Sigma)$-continuity of $U_{\sigma \in \Sigma}(t, \tau)$, we can get that

$$
\left(w_{k_{l}}(t), m_{k_{l}}(t)\right) \rightarrow U_{\sigma^{*}}(t, \tau)(v, p)=(w, m) \quad \text { in } E_{0}, \forall t>\tau
$$

From (119), we can see that the solution trajectory $\left(w_{k_{l}}(t), m_{k_{l}}(t)\right)$ is created by $U_{T\left(t_{k_{l}}-h-\tau\right) \sigma}(t+\tau, \tau)$ starting at $U_{\sigma}\left(t_{k_{l}}-h, \tau\right)\left(w_{k_{l}}^{0}, m_{k_{l}}^{0}\right)$. By (115), (119), and (122), we have that

$$
\begin{aligned}
& \left\|w_{k_{l}}(t)\right\|_{H_{\mathrm{per}}^{2 \alpha}}^{2}+F\left(w_{k_{l}}(t), m_{k_{l}}(t)\right) \\
& =e^{-2 \delta(t-\tau)}\left(\left\|U_{\sigma}\left(t_{k_{l}}-h, \tau\right) w_{k_{l}}^{0}\right\|_{H_{\mathrm{per}}^{2 \alpha}}^{2}\right. \\
& +F\left(U_{\sigma}\left(t_{k_{l}}-h, \tau\right) w_{k_{l}}^{0}, U_{\sigma}\left(t_{k_{l}}-h, \tau\right.\right. \\
& +\int_{\tau}^{t} e^{-2 \delta(t-\tau)} G\left(w_{k_{l}}(s), m_{k_{l}}(s)\right) d s \\
& =e^{-2 \delta(t-\tau)}\left(\left\|U_{\sigma}\left(t_{k_{l}}-h, \tau\right) w_{k_{l}}^{0}\right\|_{H_{\mathrm{per}}^{2 \alpha}}^{2}+F(v, p)\right) \\
& +\int_{\tau}^{t} e^{-2 \delta(t-\tau)} G\left(U_{\sigma^{*}}(s+\tau, \tau)(v, p)\right) d s .
\end{aligned}
$$$$
\left.+F\left(U_{\sigma}\left(t_{k_{l}}-h, \tau\right) w_{k_{l}}^{0}, U_{\sigma}\left(t_{k_{l}}-h, \tau\right) m_{k_{l}}^{0}\right)\right)
$$ 
Let $t=h$ in (122). Since $F$ and $G$ are weakly continuous in $E_{0}$, $\left\|U_{\sigma}\left(t_{k_{l}}-h, \tau\right)\left(w_{k_{l}}^{0}, m_{k_{l}}^{0}\right)\right\|_{H_{\mathrm{per}}^{2 \alpha}}^{2} \leqslant C$, and the Lebesgue dominated convergence theorem, we can obtain that

$$
\begin{gathered}
\limsup _{k_{l} \rightarrow \infty}\left\|U_{\sigma}\left(t_{k_{l}}, \tau\right) w_{k_{l}}^{0}\right\|_{H_{\mathrm{per}}^{2 \alpha}}^{2}+F\left(U_{\sigma^{*}}(h+\tau, \tau)(v, p)\right) \\
\leqslant e^{-2 \delta(h-\tau)}(C+F(v, p)) \\
\quad+\int_{\tau}^{h} e^{-2 \delta(h-\tau)} G\left(U_{\sigma^{*}}(s+\tau, \tau)(\nu, p)\right) d s .
\end{gathered}
$$

Since $(w, m)=U_{\sigma^{*}}(S+\tau, \tau)(v, p)$, we can see the solution $(w, m)$ as at $h$ corresponding to the initial data $(v, p)$ and the symbol $\sigma^{*}$. Similarly to (122), we have

$$
\begin{aligned}
& \|w\|_{H_{\mathrm{per}}^{2 \alpha}}^{2}+F(w, m) \\
& =e^{-2 \delta(h-t)}\left(\|v\|_{H_{\mathrm{per}}^{2 \alpha}}^{2}+F(v, p)\right) \\
& \quad+\int_{\tau}^{h} e^{-2 \delta(h-t)} G\left(U_{\sigma^{*}}(s+\tau, \tau)(v, p)\right) d s .
\end{aligned}
$$

Deducting (125) from (124), we can get that

$$
\begin{aligned}
& \limsup _{k_{l} \rightarrow \infty}\left\|U_{\sigma}\left(t_{k_{l}}, \tau\right) w_{k_{l}}^{0}\right\|_{H_{\mathrm{per}}^{2 \alpha}}^{2} \\
& \leqslant\|w\|_{H_{\mathrm{per}}^{2 \alpha}}^{2}+C e^{-2 \delta(h-t)}-e^{-2 \delta(h-t)}\|v\|_{H_{\mathrm{per}}^{2 \alpha}}^{2} \\
& \leqslant\|w\|_{H_{\mathrm{per}}^{2 \alpha}}^{2}+C e^{-2 \delta(h-t)} .
\end{aligned}
$$

As $h \rightarrow \infty$, we can get that

$$
\underset{k_{l} \rightarrow \infty}{\limsup }\left\|U_{\sigma}\left(t_{k_{l}}, \tau\right) w_{k_{l}}^{0}\right\|_{H_{\mathrm{per}}^{2 \alpha}}^{2} \leqslant\|w\|_{H_{\mathrm{per}}^{2 \alpha}}^{2} .
$$

On the other hand, the weak convergence $U_{\sigma}\left(t_{k}, \tau\right) w_{k}^{0} \rightarrow w$ implies that

$$
\liminf _{k \rightarrow \infty}\left\|U_{\sigma}\left(t_{k}, \tau\right) w_{k}^{0}\right\|_{H_{\text {per }}^{2 \alpha}}^{2} \geqslant\|w\|_{H_{\text {per }}^{2 \alpha}}^{2} .
$$

From the above two inequalities, we get that

$$
\lim _{k \rightarrow \infty}\left\|U_{\sigma}\left(t_{k}, \tau\right) w_{k}^{0}\right\|_{H_{\mathrm{per}}^{2 \alpha}}^{2}=\|w\|_{H_{\mathrm{per}}^{2 \alpha}}^{2} .
$$

Similarly to the above arguments, by using (116) we can derive that

$$
\lim _{k \rightarrow \infty}\left\|U_{\sigma}\left(t_{k}, \tau\right) m_{k}^{0}\right\|_{H_{\mathrm{per}}^{1}}^{2}=\|m\|_{H_{\mathrm{per}}^{1}}^{2}
$$

Then we get that $U_{\sigma}\left(t_{k}, \tau\right)\left(w_{k}^{0}, m_{k}^{0}\right) \rightarrow(w, m)$ in $E_{0}$. We complete the proof of the theorem.

\section{Conflict of Interests}

The authors declare that they have no conflict of interests regarding the publication of this paper.

\section{Acknowledgments}

This work was supported by the NSF of China (nos. 11371183 and 11271050) and the NSF of Shandong Province (no. ZR2013AM004).

\section{References}

[1] B. Guo, "The global solution for one class of the system of LS nonlinear wave interaction," Journal of Mathematical Research and Exposition, vol. 7, no. 1, pp. 69-76, 1987.

[2] B. Guo, "The periodic initial value problems and initial value problems for one class of generalized long-short type equations," Journal of Engineering Mathematics, vol. 8, pp. 47-53, 1991.

[3] X. Du and B. Guo, "The global attractor for LS type equation in $\mathbb{R}^{1}$, , Acta Mathematicae Applicatae Sinica, vol. 28, pp. 723-734, 2005.

[4] Y. Li, "Long time behavior for the weakly damped driven longwave-short-wave resonance equations," Journal of Differential Equations, vol. 223, no. 2, pp. 261-289, 2006.

[5] R.-F. Zhang, "Existence of global attractor for LS type equations," Journal of Mathematical Research and Exposition, vol. 26, no. 4, pp. 708-714, 2006.

[6] H. Cui, J. Xin, and A. Li, "Weakly compact uniform attractor for the nonautonomous long-short wave equations," Abstract and Applied Analysis, vol. 2013, Article ID 601325, 13 pages, 2013.

[7] R. P. Feynman and A. R. Hibbs, Quantum Mechanics and Path Integrals, McGraw-Hill, New York, NY, USA, 1965.

[8] N. Laskin, "Fractional quantum mechanics and Lévy path integrals," Physics Letters A, vol. 268, no. 4-6, pp. 298-305, 2000.

[9] N. Laskin, "Fractional quantum mechanics," Physical Review E: Statistical Physics, Plasmas, Fluids, and Related Interdisciplinary Topics, vol. 62, no. 3, pp. 3135-3145, 2000.

[10] D. Baleanu, K. Diethelm, E. Scalas, and J. J. Trujillo, Fractional Calculus: Models and Numerical Methods, Series on Complexity, Nonlinearity and Chaos, World Scientific Publishing, 2012.

[11] X. Guo and M. Xu, "Some physical applications of fractional Schrödinger equation," Journal of Mathematical Physics, vol. 47, no. 8, Article ID 082104, 2006.

[12] D. Baleanu and A. K. Golmankhaneh, "Solving of the fractional non-linear and linear Schrödinger equations by homotopy perturbation method," Romanian Journal of Physics, vol. 54, no. 10, pp. 823-832, 2009.

[13] R. Eid, S. I. Muslih, D. Baleanu, and E. Rabei, "On fractional Schrödinger equation in $\alpha$-dimensional fractional space," Nonlinear Analysis: Real World Applications, vol. 10, no. 3, pp. 12991304, 2009.

[14] B. Guo, Y. Han, and J. Xin, "Existence of the global smooth solution to the period boundary value problem of fractional nonlinear Schrödinger equation," Applied Mathematics and Computation, vol. 204, no. 1, pp. 468-477, 2008.

[15] O. Goubet, "Regularity of the attractor for a weakly damped nonlinear Schrödinger equation in $\mathbb{R}^{2}$," Advances in Differential Equations, vol. 3, no. 3, pp. 337-360, 1998.

[16] V. V. Chepyzhov and M. I. Vishik, "Attractors of nonautonomous dynamical systems and their dimension," Journal de Mathématiques Pures et Appliquées, vol. 73, no. 3, pp. 279-333, 1994.

[17] J. Bergh and J. Laofstraom, Interpolation Spaces, Springer, Berlin, Germany, 1976. 
[18] V. Chepyzhov and M. Vishik, "Non-autonomous evolutionary equations with translation-compact symbols and their attractors," Comptes Rendus de l'Académie des Sciences Série I: Mathématique, vol. 321, no. 2, pp. 153-158, 1995.

[19] J. M. Ball, "Global attractors for damped semilinear wave equations," Discrete and Continuous Dynamical Systems Series A, vol. 10, no. 1-2, pp. 31-52, 2004.

[20] B. Guo, Infinite Dimensional Dynamical Systems, National Defense Industry Press, Beijing, China, 1st edition, 2000.

[21] J. Bergh and J. Löfström, Interpolation Spaces, Springer, Berlin, Germany, 1976.

[22] J. Xin, B. Guo, Y. Han, and D. Huang, "The global solution of the $(2+1)$-dimensional long wave-short wave resonance interaction equation," Journal of Mathematical Physics, vol. 49, no. 7, Article ID 073504, 2008.

[23] B. Guo and B. Wang, "The global solution and its long time behavior for a class of generalized LS type equations," Progress in Natural Science, vol. 6, no. 5, pp. 533-546, 1996.

[24] R. Zhang and B. Guo, "Global solution and its long time behavior for the generalized long-short wave equations," Journal of Partial Differential Equations, vol. 18, no. 3, pp. 206-218, 2005. 


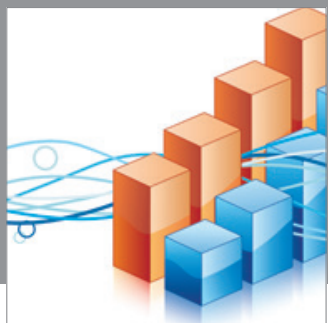

Advances in

Operations Research

mansans

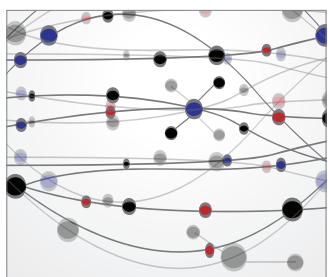

The Scientific World Journal
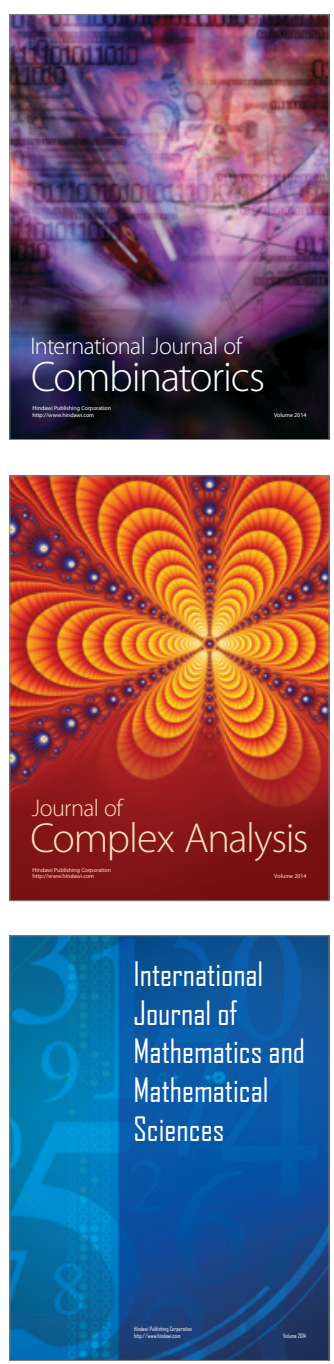
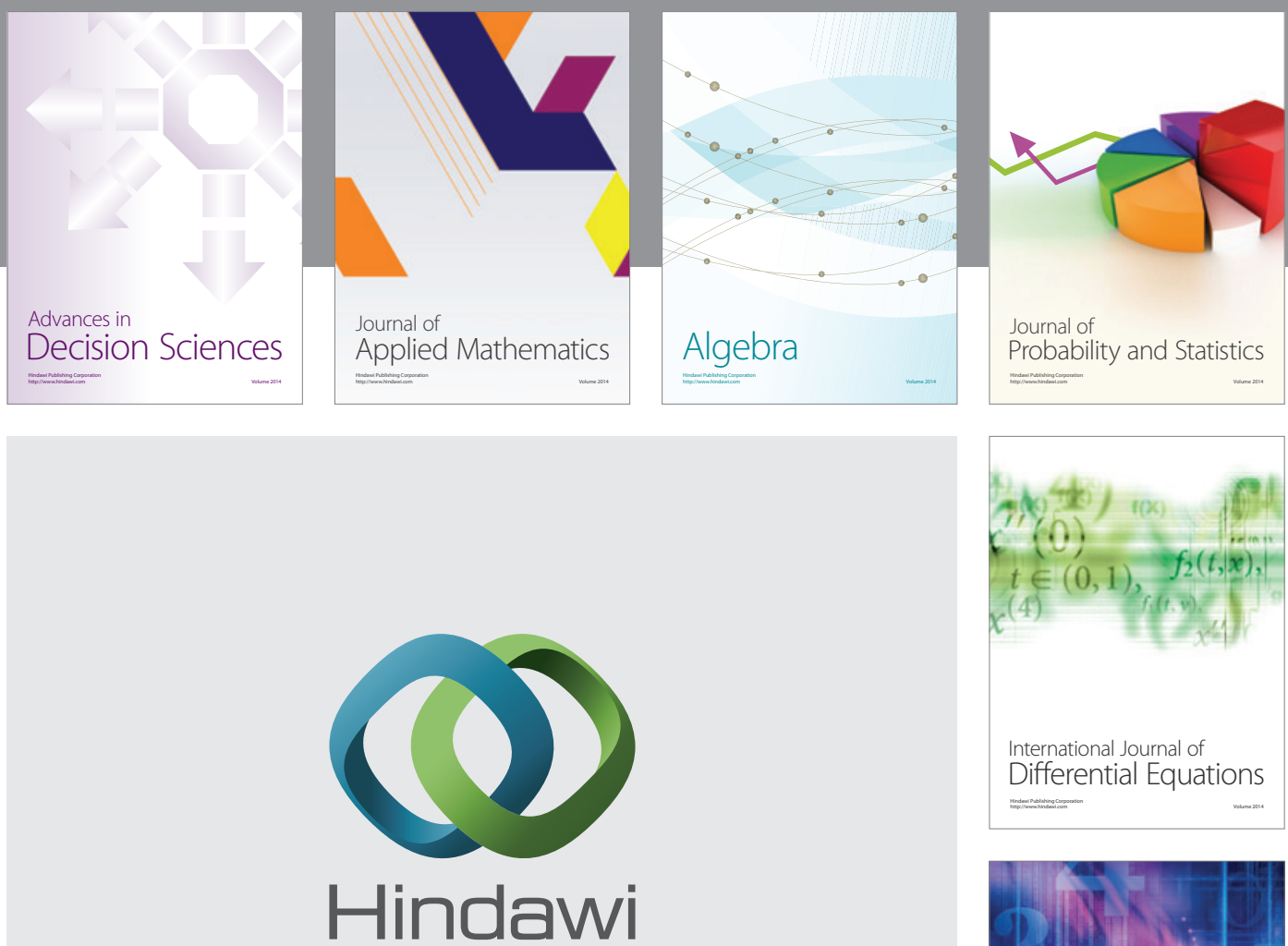

Submit your manuscripts at http://www.hindawi.com
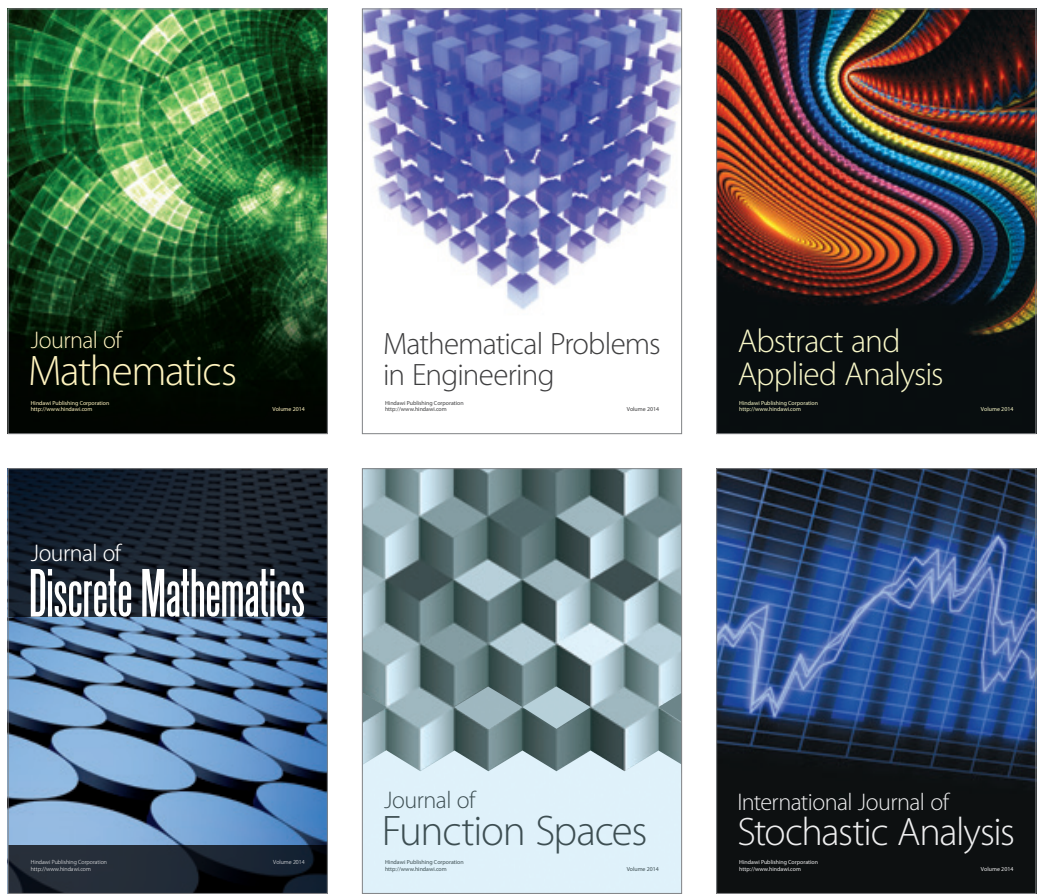

Journal of

Function Spaces

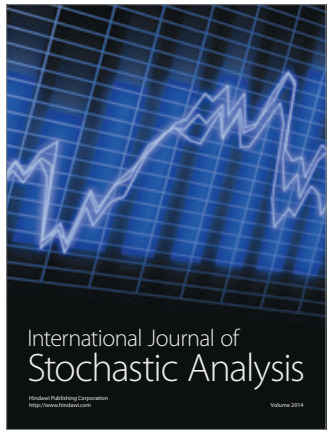

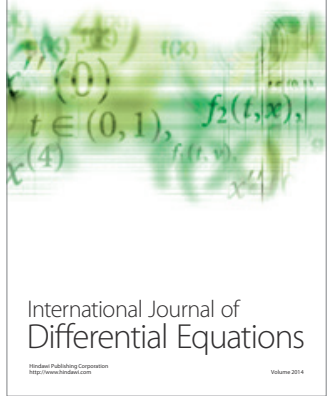
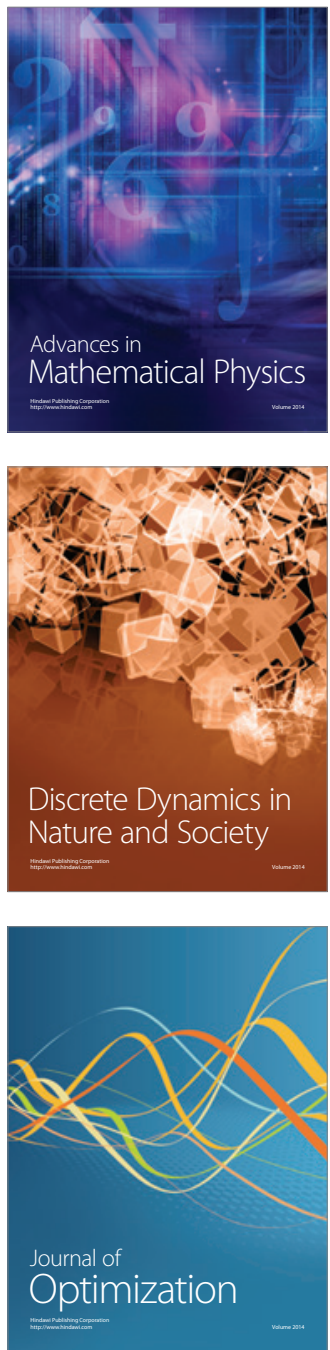